MATHEMATICS OF COMPUTATION

Volume 72, Number 243, Pages 1117-1145

S 0025-5718(03)01490-X

Article electronically published on February 7, 2003

\title{
HYBRID FINITE ELEMENT METHODS FOR THE SIGNORINI PROBLEM
}

\author{
F. BEN BELGACEM AND Y. RENARD
}

\begin{abstract}
We study three mixed linear finite element methods for the numerical simulation of the two-dimensional Signorini problem. Applying Falk's Lemma and saddle point theory to the resulting discrete mixed variational inequality allows us to state the convergence rate of each of them. Two of these finite elements provide optimal results under reasonable regularity assumptions on the Signorini solution, and the numerical investigation shows that the third method also provides optimal accuracy.
\end{abstract}

\section{INTRODUCTION AND FUNCTIONAL TOOLS}

Linear finite elements are currently used for the approximation of contact problems with unilateral Signorini boundary conditions. Developing efficient computing tools for the numerical simulation of such models is of a permanent growing interest in many physical fields: hydrostatics, thermics, solid mechanics, etc., (we refer to [17] and to [25] for a large review of the main unilateral contact models). The particular feature of the unilateral problems is that the mathematical variational statement leads to variational inequalities set on closed convex functional cones. The modeling of the nonpenetration condition in the discrete finite element level is of crucial importance. This condition may be imposed stricto-sensus on the displacement or relaxed and expressed in a weaker sense. On the way it is enforced depends the well posedness of the discrete inequalities and the accuracy of the approximation algorithms. This point is addressed in many works especially for Lagrangian finite element discretizations (see 19], 24, [21, [33, 22], 3], 26]), where different modelings are suggested and studied under a variational formulation where the displacement is the only unknown of the problem, or under a mixed variational formulation where the displacement and the stress on the contact zone are independent unknowns. Their numerical analysis established more or less satisfactory asymptotic convergence rates. For instance, when the frictionless Signorini problem is considered, imposing the nonpenetration in a strong way on the displacement field - assumed to be of class $H^{2}$ (the standard Sobolev space of degree 2 ) - the convergence rate is known to decay like $C h^{\frac{3}{4}}$ with respect to the energy norm, $h$ being the mesh size (see [24, 22]). The convergence can be improved under some reasonable assumptions, and the error estimate is reduced to $C h \sqrt{|\log h|}$ in 3]. An alternative consists in enforcing the nonpenetration in some weaker sense,

Received by the editor March 1, 2000 and, in revised form, November 28, 2001.

2000 Mathematics Subject Classification. Primary 35J85, 73J05.

Key words and phrases. Variational inequalities, mixed formulation, finite element approximation, bubble-stabilization. 
as proposed in [23], which leads to a convergence rate of order $C h^{\frac{1}{4}}$. Despite the poor convergence rate proven therein - it can be actually squared to $C h^{\frac{1}{2}}$, see section 4 - this approach has the attractive feature that the stress computed on the contact zone has the good sign; in addition, the numerical error decays like $h$ in practice (see Section 7). In [26], another weak modeling of the unilateral condition is proposed which yields a (theoretically) faster decaying of the error, of order $C h^{\frac{3}{4}}$. However, the main drawback is that the approximation method is coherent only when none of the extremities of the contact zone is subjected to a prescribed displacement (Dirichlet boundary condition); otherwise the discretization involves some spurious modes and the problem is then ill-posed. Moreover, it could hardly be extended to three-dimensional problems, where the number of spurious modes grows drastically.

In the present work, each of those finite element methods is revisited. They will all be expressed in a mixed formulation. The asymptotic convergence rates on the displacement of the first two discretizations are improved, and an error estimate on the Lagrange multiplier is stated (sections 4 and 5). Section 6 is dedicated to a stabilization of the third algorithm by bubble functions, which makes it extensible to general circumstances with a quasi-optimal error estimate and renders it conceptually coherent in three dimensions with an optimal accuracy (see [5]). In the first sections we lay a mathematical framework for the variational direct and mixed formulations of the exact Signorini problem (section 2), and we describe the finite element framework needed here (section 3). A numerical investigation of the three methods is described in section 7 , in order to show the reliability of each of them.

Notation. We need to set some notation and to recall some functional tools necessary to our analysis. Let $\Omega \subset \mathbb{R}^{2}$ be a Lipschitz domain with generic point $\boldsymbol{x}$. The Lebesgue space $L^{p}(\Omega)$ is endowed with the norm: $\forall \psi \in L^{p}(\Omega)$,

$$
\|\psi\|_{L^{p}(\Omega)}=\left(\int_{\Omega}|\psi(\boldsymbol{x})|^{p} d \boldsymbol{x}\right)^{\frac{1}{p}}
$$

We make constant use of the standard Sobolev space $H^{m}(\Omega), m \geq 1$, provided with the norm

$$
\|\psi\|_{H^{m}(\Omega)}=\left(\sum_{0 \leq|\alpha| \leq m}\left\|\partial^{\alpha} \psi\right\|_{L^{2}(\Omega)}^{2}\right)^{\frac{1}{2}},
$$

where $\alpha=\left(\alpha_{1}, \alpha_{2}\right)$ is a multi-index in $\mathbb{N}^{2}$ and the symbol $\partial^{\alpha}$ represents a partial derivative $\left(H^{0}(\Omega)=L^{2}(\Omega)\right)$. The fractional order Sobolev space $H^{\nu}(\Omega), \nu \in \mathbb{R}_{+} \backslash \mathbb{N}$, is defined by the norm

$$
\|\psi\|_{H^{\nu}(\Omega)}=\left(\|\psi\|_{H^{m}(\Omega)}^{2}+\sum_{|\alpha|=m} \int_{\Omega} \int_{\Omega} \frac{\left(\partial^{\alpha} \psi(\boldsymbol{x})-\partial^{\alpha} \psi(\boldsymbol{y})\right)^{2}}{|\boldsymbol{x}-\boldsymbol{y}|^{2+2 \theta}} d \boldsymbol{x} d \boldsymbol{y}\right)^{\frac{1}{2}},
$$

where $\nu=m+\theta, m$ is the integer part of $\nu$ and $\theta \in] 0,1[$ is the decimal part (see [1], 20]). The closure in $H^{\nu}(\Omega)$ of $\mathscr{D}(\Omega)$ is denoted $H_{0}^{\nu}(\Omega)$, where $\mathscr{D}(\Omega)$ is the space of infinitely differentiable functions with support in $\Omega$.

For any portion $\gamma$ of the boundary $\partial \Omega$, the Hilbert space $H^{\frac{1}{2}}(\gamma)$ is associated with the norm

$$
\|\psi\|_{H^{\frac{1}{2}}(\gamma)}=\left(\|\psi\|_{L^{2}(\gamma)}^{2}+\int_{\gamma} \int_{\gamma} \frac{(\psi(\boldsymbol{x})-\psi(\boldsymbol{y}))^{2}}{|\boldsymbol{x}-\boldsymbol{y}|^{2}} d \Gamma d \Gamma\right)^{\frac{1}{2}} .
$$


The space $H^{-\frac{1}{2}}(\gamma)$ is the dual space of $H^{\frac{1}{2}}(\gamma)$, and the duality pairing is denoted $\langle., .\rangle_{\frac{1}{2}, \gamma}$. The special space $H_{00}^{\frac{1}{2}}(\gamma)$ is defined as the set of the restrictions to $\gamma$ of the functions of $H^{\frac{1}{2}}(\partial \Omega)$ that vanish on $\partial \Omega \backslash \gamma$; it is also obtained by Hilbert interpolation between $H_{0}^{1}(\gamma)$ and $L^{2}(\gamma)$.

Sometimes, we need to use the Hölder space $\mathscr{C}^{0, \alpha}(\gamma), 0<\alpha \leq 1$, defined as

$$
\mathscr{C}^{0, \alpha}(\gamma)=\left\{\psi \in \mathscr{C}^{0}(\gamma), \quad\|\psi\|_{\mathscr{C}^{0, \alpha}(\gamma)}<\infty\right\},
$$

where

$$
\|\psi\|_{\mathscr{C}^{0, \alpha}(\gamma)}=\sup _{\boldsymbol{x} \in \gamma}|\psi(\boldsymbol{x})|+\sup _{\boldsymbol{x}, \boldsymbol{y} \in \gamma} \frac{|\psi(\boldsymbol{x})-\psi(\boldsymbol{y})|}{|\boldsymbol{x}-\boldsymbol{y}|^{\alpha}} .
$$

\section{The Continuous Setting of Signorini's Problem}

Let $\Omega$ be a Lipschitz bounded domain in $\mathbb{R}^{2}$. The boundary $\partial \Omega$ is a union of three nonoverlapping portions $\Gamma_{u}, \Gamma_{g}$ and $\Gamma_{C}$. The vertices of $\Gamma_{C}$ are $\left\{\boldsymbol{c}_{1}, \boldsymbol{c}_{2}\right\}$ and those of $\Gamma_{u}$ are $\left\{\boldsymbol{c}_{1}^{\prime}, \boldsymbol{c}_{2}^{\prime}\right\}$. The part $\Gamma_{u}$ of nonzero measure is subjected to Dirichlet conditions, while on $\Gamma_{g}$ a Neumann condition is prescribed, and $\Gamma_{C}$ is the candidate to be in contact with a rigid obstacle.

For given data $f \in L^{2}(\Omega)$ and $g \in H^{-\frac{1}{2}}\left(\Gamma_{g}\right)$, the Signorini problem consists in finding $u$ such that

$$
\begin{aligned}
& -\Delta u=f \quad \text { in } \Omega \text {, } \\
& u=0 \quad \text { on } \Gamma_{u}, \\
& \frac{\partial u}{\partial \boldsymbol{n}}=g \quad \text { on } \Gamma_{g}, \\
& u \geq 0, \quad \frac{\partial u}{\partial \boldsymbol{n}} \geq 0, \quad u \frac{\partial u}{\partial \boldsymbol{n}}=0 \quad \text { on } \Gamma_{C},
\end{aligned}
$$

where $\boldsymbol{n}$ is the outward unit normal to $\partial \Omega$. Most often, the modeling of the contact condition is formulated using a gap function $\alpha$ defined on $\Gamma_{C}$, so that instead of $u \geq 0$ and the saturation condition $u \frac{\partial u}{\partial \boldsymbol{n}}=0$ we have $u-\alpha \geq 0$ and $(u-\alpha) \frac{\partial u}{\partial \boldsymbol{n}}=$ 0 on the contact zone $\Gamma_{C}$ (see [17]). As the whole subsequent analysis can be extended straightforwardly to the case where $\alpha$ does not vanish, we choose, only for conciseness, to take $\alpha=0$.

Remark 2.1. Many important applications of Signorini's problem can be found in physical or mechanical fields. For instance, in hydrostatics, consider a fluid contained in a region $\Omega$ limited partly by a thin membrane $\Gamma_{C}$ which is semipermeable, meaning that it allows the fluid to pass through only in one directiononly to get in $\Omega$. When subjected to an external pressure $\alpha_{\mid \Gamma_{C}}$, the internal pressure field $p$ is a solution of equations (2.1)-(2.4) Another example comes from the air conditioning field, the temperature distribution $u$ within a room heated by a wall device, which injects a heat flux only when $u$ drops below a threshold so that $u$ is permanently maintained higher than a fixed temperature $\alpha$ in the vicinity of the device, is modeled by equations (2.1)-(2.4). The last situation that we shall consider is taken from deformable solid mechanics. The displacement of a body $\Omega$ supported by a frictionless rigid foundation $\Gamma_{C}$, fixed along a part $\Gamma_{\boldsymbol{u}}$ of the border and subjected to external forces $\boldsymbol{f}_{\mid \Omega}$ and $\boldsymbol{g}_{\mid \Gamma_{\boldsymbol{g}}}$, is a solution to the following problem 


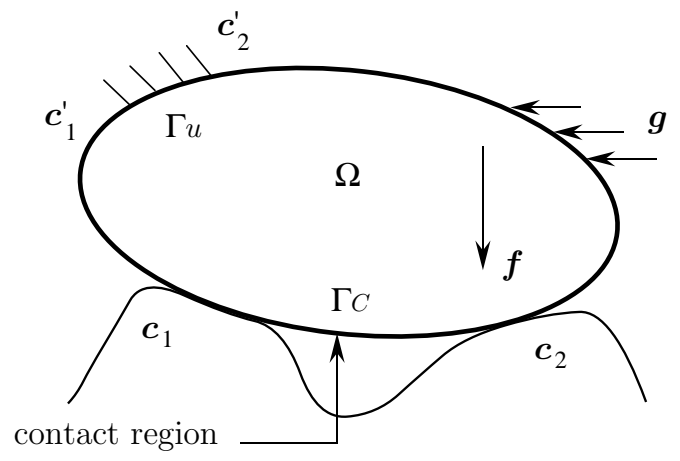

FiguRE 2.1.

(see Figure 2.1):

$$
\begin{aligned}
-\boldsymbol{d i v} \sigma(\boldsymbol{u}) & =\boldsymbol{f} & & \text { in } \Omega, \\
\sigma(\boldsymbol{u}) \boldsymbol{n} & =\boldsymbol{g} & & \text { on } \Gamma_{\boldsymbol{g}} \\
\boldsymbol{u} & =0 & & \text { on } \Gamma_{\boldsymbol{u}} .
\end{aligned}
$$

The bold symbol $\boldsymbol{d i v}$ denotes the divergence operator of a tensor function and is defined as $\boldsymbol{d i v} \sigma=\left(\frac{\partial \sigma_{i j}}{\partial x_{j}}\right)_{i}$. The stress tensor is obtained from the displacement through the constitutive law $\sigma(\boldsymbol{u})=A(\boldsymbol{x}) \varepsilon(\boldsymbol{u})$, where $A(\boldsymbol{x}) \in\left(L^{\infty}(\Omega)\right)^{16}$; the Hooke tensor is of fourth order, symmetric and elliptic.

Finally, to close the system, frictionless contact conditions are needed on $\Gamma_{C}$. Denoting by $\sigma_{\boldsymbol{n}}$ the normal component of $(\sigma \boldsymbol{n})$ and by $\sigma_{\boldsymbol{t}}$ its tangential component, the contact conditions are formulated as follows:

$$
\begin{aligned}
\boldsymbol{u} . \boldsymbol{n} \leq 0, \quad \sigma_{\boldsymbol{n}} & \leq 0, \quad \sigma_{\boldsymbol{n}}(\boldsymbol{u} . \boldsymbol{n})=0, \\
\sigma_{\boldsymbol{t}} & =0 .
\end{aligned}
$$

In the linear elasticity context, where the body undergoes small displacements the strain tensor is $\varepsilon(\boldsymbol{u})=\frac{1}{2}\left(\nabla \boldsymbol{u}+(\nabla \boldsymbol{u})^{T}\right)$, problem (2.5)-(2.8) has the same properties as the Signorini problem (2.1)-(2.4) we intend to study in detail. Then, the whole numerical analysis is extended also to the unilateral contact elastic problem.

The functional framework well suited to solve Signorini's problem consists in working with the subspace $H_{0}^{1}\left(\Omega, \Gamma_{u}\right)$ of $H^{1}(\Omega)$ made of functions that vanish at $\Gamma_{u}$, endowed with the semi-norm which is equivalent, by Poincaré's inequality, to the norm of $H^{1}(\Omega)$. Accounting for the unilateral contact condition on $\Gamma_{C}$ in the weak formulation may be done in two different ways. In the first, the contact condition enters the variational formulation through the introduction of the closed convex set

$$
K(\Omega)=\left\{v \in H_{0}^{1}\left(\Omega, \Gamma_{u}\right), \quad v_{\mid \Gamma_{C}} \geq 0, \text { a.e. }\right\} .
$$

The primal variational principle for Signorini's problem produces the variational inequality: find $u \in K(\Omega)$ such that

$$
a(u, v-u) \geq L(v-u), \quad \forall v \in K(\Omega) .
$$


In (2.9) we have set

$$
\begin{aligned}
a(u, v) & =\int_{\Omega} \nabla u \nabla v d \boldsymbol{x}, \\
L(v) & =\int_{\Omega} f v d \boldsymbol{x}+\langle g, v\rangle_{\frac{1}{2}, \Gamma_{g}} .
\end{aligned}
$$

Applying Stampacchia's theorem (see [25, 22]), the weak problem (2.9) is well posed and has only one solution in $K(\Omega)$ that depends continuously on the data $(f, g)$.

Remark 2.2. When no more regularity than $H^{1}(\Omega)$ is available on the solution $u$, the normal derivative $\frac{\partial u}{\partial \boldsymbol{n}}$ does exist in $H^{-\frac{1}{2}}(\partial \Omega)$ since $\Delta u \in L^{2}(\Omega)$, but cannot be expressed pointwise. A little care should be paid to give a mathematical sense to conditions (2.3) and (2.4), which are then formulated as follows:

$$
\begin{aligned}
& \left\langle\frac{\partial u}{\partial \boldsymbol{n}}, v\right\rangle_{\frac{1}{2}, \partial \Omega}-\langle g, v\rangle_{\frac{1}{2}, \Gamma_{g}} \geq 0, \quad \forall v \in H_{00}^{\frac{1}{2}}\left(\partial \Omega, \Gamma_{u}\right), \quad v_{\mid \Gamma_{C}} \geq 0, \\
& \left\langle\frac{\partial u}{\partial \boldsymbol{n}}, u\right\rangle_{\frac{1}{2}, \partial \Omega}-\langle g, u\rangle_{\frac{1}{2}, \Gamma_{g}}=0,
\end{aligned}
$$

where $H_{00}^{\frac{1}{2}}\left(\partial \Omega, \Gamma_{u}\right)$ is the subspace of $H^{\frac{1}{2}}(\partial \Omega)$ of the functions that vanish on $\Gamma_{u}$. Roughly, (2.10) says that $\frac{\partial u}{\partial \boldsymbol{n}}=g$ on $\Gamma_{g}$ and $\frac{\partial u}{\partial \boldsymbol{n}} \geq 0$ on $\Gamma_{C}$, while (2.11) expresses the saturation condition $u \frac{\partial u}{\partial \boldsymbol{n}}=0$ on $\Gamma_{C}$.

A second weak formulation is obtained from writing the solution $u$ as the minimizing argument of the energy functional under the unilateral contact inequality constraints $u_{\mid \Gamma_{C}} \geq 0$. Such a problem can be reformulated as a saddle point problem, and hence $u$ is the first component of the saddle point $(u, \varphi)$ of the Lagrangian

$$
\mathcal{L}(v, \psi)=\frac{1}{2} a(v, v)-L(v)-b(\psi, v)
$$

defined on $H_{0}^{1}\left(\Omega, \Gamma_{u}\right) \times M\left(\Gamma_{C}\right)$, where

$$
M\left(\Gamma_{C}\right)=\left\{\psi \in H^{-\frac{1}{2}}\left(\Gamma_{C}\right), \quad \psi \geq 0\right\} .
$$

The nonnegativity of a distribution $\psi \in H^{-\frac{1}{2}}\left(\Gamma_{C}\right)$ is to be understood in the sense that $\langle\psi, \chi\rangle_{\frac{1}{2}, \Gamma_{C}} \geq 0$ for any $\chi \in H^{\frac{1}{2}}\left(\Gamma_{C}\right), \chi \geq 0$. In (2.12) we have denoted

$$
b(\psi, v)=\langle\psi, v\rangle_{\frac{1}{2}, \Gamma_{C}} .
$$

The properties of $\mathcal{L}$ allow us to deduce that the saddle point $(u, \varphi)$ is a solution of the mixed variational system: find $(u, \varphi) \in H_{0}^{1}\left(\Omega, \Gamma_{u}\right) \times M\left(\Gamma_{C}\right)$ such that

$$
\begin{aligned}
a(u, v)-b(\varphi, v) & =L(v), \quad \forall v \in H_{0}^{1}\left(\Omega, \Gamma_{u}\right), \\
b(\psi-\varphi, u) & \geq 0, \quad \forall \psi \in M\left(\Gamma_{C}\right) .
\end{aligned}
$$

A complete analysis of this mixed problem is provided in [24], Theorem 3.14 (see also [22], where an existence and uniqueness result is proven. Besides, we have the following stability:

$$
\|u\|_{H^{1}(\Omega)}+\|\varphi\|_{H^{-\frac{1}{2}\left(\Gamma_{C}\right)}} \leq C\left(\|f\|_{L^{2}(\Omega)}+\|g\|_{H^{-\frac{1}{2}\left(\Gamma_{g}\right)}}\right) .
$$


Remark 2.3. By the Hahn-Banach theorem it can be stated that

$$
K(\Omega)=\left\{v \in H_{0}^{1}\left(\Omega, \Gamma_{u}\right), \quad b(\psi, v) \geq 0, \forall \psi \in M\left(\Gamma_{C}\right)\right\} .
$$

It is easy to show that if $(u, \varphi)$ is a solution of (2.13)-(2.14), then $u \in K(\Omega)$ and satisfies (2.10) and (2.11). Therefore, it can be proven that $u$ is also a solution of (2.9). Conversely, thanks to the existence and uniqueness results for both problems, if $u$ is the solution of (2.9), then $(u, \varphi)$ is the solution of the mixed problem (2.13)(2.14), where $\varphi \in H^{-\frac{1}{2}}\left(\Gamma_{C}\right)$ is such that

$$
\langle\varphi, v\rangle_{\frac{1}{2}, \Gamma_{C}}=\left\langle\frac{\partial u}{\partial \boldsymbol{n}}, v\right\rangle_{\frac{1}{2}, \partial \Omega}-\langle g, v\rangle_{\frac{1}{2}, \Gamma_{g}}, \quad \forall v \in H_{00}^{\frac{1}{2}}\left(\partial \Omega, \Gamma_{u}\right) .
$$

When $u$ is sufficiently regular $\left(u \in H^{\sigma}(\Omega), \sigma>\frac{3}{2}\right)$, the normal derivative $\frac{\partial u}{\partial \boldsymbol{n}}$ can be defined pointwise; hence the function $\varphi$ coincides with $\frac{\partial u}{\partial \boldsymbol{n}} \mid \Gamma_{C}$, and $b(\varphi, u)=0$.

\section{LINEAR FINITE ELEMENT FRAMEWORK}

We shall now describe the finite elements used in the approximation of the mixed problem (2.13)-(2.14) and of the variational inequality (2.9). The shape of the domain $\Omega$ is assumed polygonal, so that it can be exactly covered by rectilinear finite elements. For any given discretization parameter $h>0$, let $\mathscr{T}_{h}$, a partition of $\Omega$ into triangles (or quadrangles) with a maximum size $h$, be given

$$
\bar{\Omega}=\bigcup_{\kappa \in \mathscr{T}_{h}} \bar{\kappa} .
$$

The set of the vertices of the elements is $\Xi_{h}$. The family $\left(\mathscr{T}_{h}\right)_{h}$ is assumed to be $\mathscr{C}^{0}$-regular in the classical sense (see [13], [31]). Moreover, $\mathscr{T}_{h}$ is built in such a way that the set $\left\{\boldsymbol{c}_{1}, \boldsymbol{c}_{2}, \boldsymbol{c}_{1}^{\prime}, \boldsymbol{c}_{2}^{\prime}\right\}$ is included in $\Xi_{h}$. For any $\kappa \in \mathscr{T}_{h}, \mathcal{P}_{q}(\kappa)$ stands for the set of polynomials of total degree $\leq q$. Then we introduce the finite dimensional subspace $X_{h}(\Omega)$ of $H_{0}^{1}\left(\Omega, \Gamma_{u}\right)$,

$$
X_{h}(\Omega)=\left\{v_{h} \in \mathscr{C}(\bar{\Omega}), \forall \kappa \in \mathscr{T}_{h}, v_{h \mid \kappa} \in \mathcal{P}_{1}(\kappa), \quad v_{h \mid \Gamma_{u}}=0\right\} .
$$

For simplification, in particular when we are involved with fractional Sobolev norms, the contact portion $\Gamma_{C}$ is assumed to be a straight line. More general cases require higher technicalities, which are beyond the scope of this work.

Due to the $\mathscr{C}^{0}$-regularity hypothesis, $\Gamma_{C}$ inherits a regular mesh, denoted $\mathscr{T}_{h}^{C}$ characterized by the subdivision $\left(\boldsymbol{x}_{i}^{C}\right)_{0 \leq i \leq i^{*}}$ with $\boldsymbol{x}_{0}^{C}=\boldsymbol{c}_{1}$ and $\boldsymbol{x}_{i^{*}}^{C}=\boldsymbol{c}_{2}$; and $\left(t_{i}=\right] \boldsymbol{x}_{i}^{C}, \boldsymbol{x}_{i+1}^{C}[)_{0 \leq i \leq i^{*}-1}$ are its elements. Furthermore, if $\left(\mathcal{I}_{h}\right)$ denotes the standard Lagrange interpolation operator, then for any $(\mu, \nu) \in[0,1] \times] 1,2]$, the following error estimate holds (see [13]): $\forall v \in H^{\nu}(\Omega)$,

$$
\left\|v-\mathcal{I}_{h} v\right\|_{H^{\mu}(\Omega)} \leq C h^{\nu-\mu}\|v\|_{H^{\nu}(\Omega)} .
$$

The constant $C$ depends only on $(\mu, \nu, \Omega)$. Throughout this section, the approximation of the Lagrange multipliers space $M\left(\Gamma_{C}\right)$ is made by choosing a closed convex cone $M_{h}\left(\Gamma_{C}\right)$, which for the two first methods (sections 4 and 5 ) will be a subset of the trace space

$$
\begin{aligned}
W_{h}\left(\Gamma_{C}\right) & =\left\{\psi_{h}=v_{h \mid \Gamma_{C}}, \quad v_{h} \in X_{h}(\Omega)\right\} \\
& =\left\{\psi_{h} \in \mathscr{C}\left(\bar{\Gamma}_{C}\right), \quad \forall t \in \mathscr{T}_{h}^{C}, \quad \psi_{h \mid t} \in \mathcal{P}_{1}(t)\right\},
\end{aligned}
$$


containing the "nonnegative" functions. At this level notion of the nonnegativity is not yet determined. It may have different meanings according to the mixed finite elements used, and needs to be made more precise later on.

The discrete framework being adopted, the discrete mixed problem is obtained from (2.13)-(2.14) by a standard Galerkin procedure and consists in finding $\left(u_{h}, \varphi_{h}\right)$ $\in X_{h}(\Omega) \times M_{h}\left(\Gamma_{C}\right)$ such that

$$
\begin{aligned}
a\left(u_{h}, v_{h}\right)-b\left(\varphi_{h}, v_{h}\right) & =L\left(v_{h}\right), \quad \forall v_{h} \in X_{h}(\Omega), \\
b\left(\psi_{h}-\varphi_{h}, u_{h}\right) & \geq 0, \quad \forall \psi_{h} \in M_{h}\left(\Gamma_{C}\right) .
\end{aligned}
$$

Proving that this problem has only one solution $\left(u_{h}, \varphi_{h}\right) \in X_{h}(\Omega) \times M_{h}\left(\Gamma_{C}\right)$ is an easy matter using tools of convex optimization in finite dimensions. However, in order to derive optimal stability results with respect to the natural norms, $H^{1}(\Omega)$ for $u$ and $H^{-\frac{1}{2}}\left(\Gamma_{C}\right)$ for $\varphi$, it is necessary to have a uniform inf-sup condition on the bilinear form $b(.,$.$) set on M_{h}\left(\Gamma_{C}\right) \times X_{h}(\Omega)$. This inf-sup condition is strongly connected to the continuity of the $L^{2}\left(\Gamma_{C}\right)$-orthogonal projection $\pi_{h}$ on $W_{h}\left(\Gamma_{C}\right)$, restricted to $H^{\frac{1}{2}}\left(\Gamma_{C}\right)$ with $\left\|\pi_{h}\right\|_{\mathcal{L}\left(H^{\frac{1}{2}}\left(\Gamma_{C}\right)\right)}$ independent of $h$. Such a result is obvious when the one dimensional mesh $\mathscr{T}_{h}^{C}$ is quasi-uniform, and this allows us to use the inverse inequality. The quasi-uniformity assumption is particularly stringent and does not authorize some "interesting" meshing used in adaptive processes for instance. It has been weakened, thanks to the work of Crouzeix and Thomée (see [16]), which allows for a larger class of meshes. The triangulation $\mathscr{T}_{h}^{C}$ is said to satisfy the Crouzeix-Thomée criterion if

$$
\frac{\left|t_{i}\right|}{\left|t_{j}\right|} \leq C \beta^{|i-j|}, \quad \forall i, j\left(0 \leq i, j \leq i^{*}-1\right),
$$

where $1 \leq \beta<4$ and $C$ does not depend on $h$ (the number 4 is related to the finite element degree $q=1$, i.e., $\left.\beta<(q+1)^{2}\right)$. Observe that a wide class of radical and geometric meshes fulfill this criterion. Under the assumption (3.4) and for any $\nu \in[0,1]$, the operator $\pi_{h}$ has the stability (see [16]): $\forall \psi \in H^{\nu}\left(\Gamma_{C}\right)$

$$
\left\|\pi_{h} \psi\right\|_{H^{\nu}\left(\Gamma_{C}\right)} \leq C\|\psi\|_{H^{\nu}\left(\Gamma_{C}\right)} .
$$

Moreover, the following approximation result holds (see [16]): for any $\nu \in[0,1]$ and $\mu \in\left[0, \nu+\frac{1}{2}\right]$ we have, $\forall \psi \in H^{\frac{1}{2}+\nu}\left(\Gamma_{C}\right)$,

$$
h^{-\frac{1}{2}}\left\|\psi-\pi_{h} \psi\right\|_{H^{-\frac{1}{2}}\left(\Gamma_{C}\right)}+h^{\mu}\left\|\psi-\pi_{h} \psi\right\|_{H^{\mu}\left(\Gamma_{C}\right)} \leq C h^{\nu+\frac{1}{2}}\|\psi\|_{H^{\frac{1}{2}+\nu}\left(\Gamma_{C}\right)} .
$$

The boundedness of the error with respect to the $H^{-\frac{1}{2}}\left(\Gamma_{C}\right)$-norm is obtained by duality and does not require any additional hypothesis on $\mathscr{T}_{h}^{C}$, while the estimate in $H^{\nu}\left(\Gamma_{C}\right)$ is a direct consequence of (3.5) and is valid under condition (3.4).

Lemma 3.1. Under the assumption (3.4) on the mesh $\mathscr{T}_{h}^{C}$ the following inf-sup condition holds:

$$
\inf _{\psi_{h} \in W_{h}\left(\Gamma_{C}\right)} \sup _{v_{h} \in X_{h}(\Omega)} \frac{b\left(\psi_{h}, v_{h}\right)}{\left\|v_{h}\right\|_{H^{1}(\Omega)}\left\|\psi_{h}\right\|_{H^{-\frac{1}{2}}\left(\Gamma_{C}\right)}} \geq \gamma
$$

The constant $\gamma>0$ and does not depend on $h$.

Proof. Let $\psi_{h} \in W_{h}\left(\Gamma_{C}\right)$. We are going to construct $v_{h} \in X_{h}(\Omega)$ satisfying

$$
b\left(\psi_{h}, v_{h}\right) \geq\left\|\psi_{h}\right\|_{H^{-\frac{1}{2}}\left(\Gamma_{C}\right)}^{2} \quad \text { and } \quad \gamma\left\|v_{h}\right\|_{H^{1}(\Omega)} \leq\left\|\psi_{h}\right\|_{H^{-\frac{1}{2}}\left(\Gamma_{C}\right)} .
$$


Let us consider the function $v \in H^{1}(\Omega)$ such that

$$
\int_{\Omega} v w d \boldsymbol{x}+\int_{\Omega} \nabla v \nabla w d \boldsymbol{x}=b\left(\psi_{h}, w\right) \quad \forall w \in H^{1}(\Omega) .
$$

The following stability inequalities hold:

$$
c_{-}\left\|\psi_{h}\right\|_{H^{-\frac{1}{2}\left(\Gamma_{C}\right)}} \leq\|v\|_{H^{1}(\Omega)} \leq c_{+}\left\|\psi_{h}\right\|_{H^{-\frac{1}{2}}\left(\Gamma_{C}\right)} .
$$

Then we set $v_{h} \in X_{h}(\Omega)$ such that $v_{h \mid \Gamma_{C}}=\pi_{h}\left(v_{\mid \Gamma_{C}}\right)$ and

$$
\left\|v_{h}\right\|_{H^{1}(\Omega)} \leq c\left\|\pi_{h} v\right\|_{H^{\frac{1}{2}\left(\Gamma_{C}\right)}} \leq c\|v\|_{H^{\frac{1}{2}\left(\Gamma_{C}\right)}} .
$$

Such a $v_{h}$ is built using the stable finite element extension operator studied in [8]. The first statement of (3.7) is valid because

$$
b\left(\psi_{h}, v_{h}\right)=b\left(\psi_{h}, v\right)=\|v\|_{H^{1}(\Omega)}^{2} \geq c_{-}^{2}\left\|\psi_{h}\right\|_{H^{-\frac{1}{2}}\left(\Gamma_{C}\right)}^{2} .
$$

The second is obtained from (3.9) and (3.10) together with the trace theorem.

This lemma implies that the bilinear form $b(.,$.$) fulfills also a uniform inf-sup$ on $M_{h}\left(\Gamma_{C}\right) \times X_{h}(\Omega)$ which yields existence and uniqueness for the mixed problem (3.2)-(3.3)

Proposition 3.2. The discrete mixed finite element problem (3.2)-(3.3) has only one solution in $X_{h}(\Omega) \times M_{h}\left(\Gamma_{C}\right)$ such that

$$
\left\|u_{h}\right\|_{H^{1}(\Omega)}+\left\|\varphi_{h}\right\|_{H^{-\frac{1}{2}}\left(\Gamma_{C}\right)} \leq C\left(\|f\|_{L^{2}(\Omega)}+\|g\|_{H^{-\frac{1}{2}}\left(\Gamma_{g}\right)}\right) .
$$

The constant $C$ does not depend on $h$.

The numerical modeling of the unilateral contact, while respecting the inclusion $M_{h}\left(\Gamma_{C}\right) \subset W_{h}\left(\Gamma_{C}\right)$, may be done in two different ways. It is possible to enforce explicitly either $u_{h} \geq 0$ on $\Gamma_{C}$ or $\varphi_{h} \geq 0$. These choices provide us with two variational problems and yield different "theoretical" convergence rates of the corresponding linear finite element approximations.

\section{MiXed Finite Elements AND "NONCONFORMinG" DISCRETIZATION OF PROBLEM (2.9)}

Sometimes, for practical reasons, it is preferred to enforce strongly the nonnegativity condition on $\varphi=\left.\frac{\partial u}{\partial \boldsymbol{n}}\right|_{C}$ rather than on $u_{\mid \Gamma_{C}}$. Then, it is explicitly incorporated in the Lagrange multipliers space

$$
M_{h}^{\varphi}\left(\Gamma_{C}\right)=\left\{\psi_{h} \in W_{h}\left(\Gamma_{C}\right), \psi_{h} \geq 0\right\} .
$$

In order to carry out the numerical analysis of this approximation let us define the discrete closed convex set

$$
K_{h}^{\varphi}(\Omega)=\left\{v_{h} \in X_{h}(\Omega), b\left(\psi_{h}, v_{h}\right) \geq 0, \forall \psi_{h} \in M_{h}^{\varphi}\left(\Gamma_{C}\right)\right\} .
$$

It is an external approximation of $K(\Omega)$, i.e., $K_{h}^{\varphi}(\Omega) \not \subset K(\Omega)$. We have $u_{h} \in$ $K_{h}^{\varphi}(\Omega)$, and from inequality (3.3) we deduce that $b\left(\varphi_{h}, u_{h}\right)=0$. By some easy manipulations we derive that $u_{h}$ is also a solution of the discrete Signorini problem: find $u_{h} \in K_{h}^{\varphi}(\Omega)$ such that

$$
a\left(u_{h}, v_{h}-u_{h}\right) \geq L\left(v_{h}-u_{h}\right), \quad \forall v_{h} \in K_{h}^{\varphi}(\Omega) .
$$

It may be viewed as a "nonconforming" approximation of the exact problem (2.9). 
An attempt at numerical analysis of this discretization is made in 23] (see also [15]). Even when the solution $u \in H^{2}(\Omega)$, the convergence estimate of order $C h^{\frac{1}{4}}$, with respect to the energy norm, is not satisfactory. Numerical evidence (see [15] and section 7) shows that the error should decay faster. The aim is to improve the numerical analysis, to recover an optimal error estimate for $u$, and to derive an error estimate for the Lagrange multiplier $\varphi$. The derivation of the error estimate for $u$ is based on an adaptation of Falk's Lemma (see [18], [24]).

Lemma 4.1. Let $u \in K(\Omega)$ be the solution of the variational Signorini inequality (2.9), and let $u_{h} \in K_{h}^{\varphi}(\Omega)$ be the solution of the discrete variational inequality (4.1). Then

$$
\begin{array}{r}
\left\|u-u_{h}\right\|_{H^{1}(\Omega)}^{2} \leq C\left[\inf _{v_{h} \in K_{h}^{\varphi}(\Omega)}\left(\left\|u-v_{h}\right\|_{H^{1}(\Omega)}^{2}+\left\langle\frac{\partial u}{\partial \boldsymbol{n}}, v_{h}\right\rangle_{\frac{1}{2}, \partial \Omega}-\left\langle g, v_{h}\right\rangle_{\frac{1}{2}, \Gamma_{g}}\right)\right. \\
\left.+\inf _{v \in K(\Omega)}\left(\left\langle\frac{\partial u}{\partial \boldsymbol{n}}, v-u_{h}\right\rangle_{\frac{1}{2}, \partial \Omega}-\left\langle g, v-u_{h}\right\rangle_{\frac{1}{2}, \Gamma_{g}}\right)\right] .
\end{array}
$$

Remark 4.2. The first infimum of the bound given in Lemma 4.1 is the approximation error, and the integral term involved there is specifically generated by the discretization of variational inequalities. The last infimum is the consistency error; it is the "variational crime" and is due to the nonconformity of the approximation.

Using Lemma 4.1, together with the finite element approximation results, we are in position to provide the convergence rate of our finite element approximation.

Theorem 4.3. Let $u \in K(\Omega)$ be the solution of the continuous Signorini problem (2.9), and $u_{h} \in K_{h}^{\varphi}(\Omega)$ the solution of (4.1). Assume $u \in H^{2}(\Omega)$. Then

$$
\left\|u-u_{h}\right\|_{H^{1}(\Omega)} \leq C h^{\frac{1}{2}}\|u\|_{H^{2}(\Omega)} .
$$

Remark 4.4. If $\Gamma_{u}$ and $\Gamma_{g}$ share a common point (as is the case in Figure 2.1), the exact solution $u$ is expected to contain a singular part that does not belong to $H^{\frac{3}{2}}$ (see [20]). However, since our goal is only to focus on the approximation behavior around $\Gamma_{C}$, we can assume that this singularity is not effective (or in an equivalent way the singular coefficient is switched off). Besides, notice that the convergence rate of the integral term depends only on the smoothness of $u$ around $\Gamma_{C}$, and unless the domain is cracked near this contact zone, the solution $u$ has a regularity exponent higher than two (see [28]), and the regularity assumed on $u$ in the theorem seems to be quiet reasonable as soon as $f$ and $g$ are sufficiently smooth.

Before giving the proof of the theorem, we need separate bounds for the approximation error and the consistency error.

Lemma 4.5. Let $u \in K(\Omega)$ be the solution of the continuous Signorini problem (2.9), and $u_{h} \in K_{h}^{\varphi}(\Omega)$ the solution of (4.1). Assume $u \in H^{2}(\Omega)$. Then

$$
\inf _{v_{h} \in K_{h}^{\varphi}(\Omega)}\left(\left\|u-v_{h}\right\|_{H^{1}(\Omega)}^{2}+\int_{\Gamma_{C}} \frac{\partial u}{\partial \boldsymbol{n}} v_{h} d \Gamma\right) \leq C h^{2}\|u\|_{H^{2}(\Omega)}^{2} .
$$

Proof. Choose $v_{h}=\mathcal{I}_{h} u+w_{h}$, with $w_{h} \in X_{h}(\Omega)$ a stable extension of the trace function $\left(\pi_{h}\left(u_{\mid \Gamma_{c}}\right)-\left(\mathcal{I}_{h} u\right)_{\mid \Gamma_{c}}\right)$. To check that $v_{h} \in K_{h}^{\varphi}(\Omega)$, it is sufficient to observe that $v_{h \mid \Gamma_{C}}=\pi_{h}\left(u_{\mid \Gamma_{c}}\right)$, and since $u_{\mid \Gamma_{c}} \geq 0$, we have, $\forall \psi_{h} \in M_{h}^{\varphi}\left(\Gamma_{C}\right)$,

$$
b\left(\psi_{h}, v_{h}\right)=\int_{\Gamma_{C}} \pi_{h}\left(u_{\mid \Gamma_{c}}\right) \psi_{h} d \Gamma=\int_{\Gamma_{C}}\left(u_{\mid \Gamma_{c}}\right) \psi_{h} d \Gamma \geq 0 .
$$


Furthermore, using (3.1) and (3.6) produces

$$
\left\|u-v_{h}\right\|_{H^{1}(\Omega)} \leq\left\|u-\mathcal{I}_{h} u\right\|_{H^{1}(\Omega)}+C\left\|\pi_{h}\left(u_{\mid \Gamma_{c}}\right)-\left(\mathcal{I}_{h} u\right)_{\mid \Gamma_{c}}\right\|_{H^{\frac{1}{2}\left(\Gamma_{C}\right)}} \leq C h\|u\|_{H^{2}(\Omega)} .
$$

In order to evaluate the integral term we use the saturation condition $\left(u \frac{\partial u}{\partial \boldsymbol{n}}\right)_{\mid \Gamma_{C}}=0$ and again the estimate (3.6):

$$
\begin{aligned}
\int_{\Gamma_{C}} \frac{\partial u}{\partial \boldsymbol{n}} v_{h} d \Gamma & =\int_{\Gamma_{C}} \frac{\partial u}{\partial \boldsymbol{n}}\left(\pi_{h} u-u\right) d \Gamma \leq\left\|\frac{\partial u}{\partial \boldsymbol{n}}\right\|_{H^{\frac{1}{2}}\left(\Gamma_{C}\right)}\left\|\pi_{h} u-u\right\|_{H^{-\frac{1}{2}}\left(\Gamma_{C}\right)} \\
& \leq C h^{2}\left\|\frac{\partial u}{\partial \boldsymbol{n}}\right\|_{H^{\frac{1}{2}}\left(\Gamma_{C}\right)}\|u\|_{H^{\frac{3}{2}\left(\Gamma_{C}\right)}} \leq C h^{2}\|u\|_{H^{2}(\Omega)}^{2} .
\end{aligned}
$$

This proves the lemma.

Lemma 4.6. Let $u \in K(\Omega)$ be the solution of the continuous Signorini problem (2.9), and $u_{h} \in K_{h}^{\varphi}(\Omega)$ the solution of (4.1). Assume $u \in H^{2}(\Omega)$. Then the consistency error is bounded as follows:

$$
\inf _{v \in K(\Omega)} \int_{\Gamma_{C}} \frac{\partial u}{\partial \boldsymbol{n}}\left(v-u_{h}\right) d \Gamma \leq C\left(h^{\frac{3}{2}}\|u\|_{H^{2}(\Omega)}+h^{\frac{1}{2}}\left\|u-u_{h}\right\|_{H^{1}(\Omega)}\right)\|u\|_{H^{2}(\Omega)} .
$$

Proof. Choose $v=u$; then we have, $\forall \psi_{h} \in M_{h}^{\varphi}\left(\Gamma_{C}\right)$,

$$
\int_{\Gamma_{C}} \frac{\partial u}{\partial \boldsymbol{n}}\left(u-u_{h}\right) d \Gamma=\int_{\Gamma_{C}}\left(\frac{\partial u}{\partial \boldsymbol{n}}-\psi_{h}\right)\left(u-u_{h}\right) d \Gamma+\int_{\Gamma_{C}} \psi_{h}\left(u-u_{h}\right) d \Gamma .
$$

Taking $\psi_{h}=r_{h}\left(\frac{\partial u}{\partial \boldsymbol{n}}\right)\left(r_{h}\right.$ is a nonstandard Clément type interpolation operator preserving nonnegativity, and is defined in Appendix A) and using (A.5), the first integral is easily handled:

$$
\int_{\Gamma_{C}}\left(\frac{\partial u}{\partial \boldsymbol{n}}-\psi_{h}\right)\left(u-u_{h}\right) d \Gamma \leq C h^{\frac{1}{2}}\|u\|_{H^{2}(\Omega)}\left\|u-u_{h}\right\|_{H^{1}(\Omega)} .
$$

Optimality is not reached in (4.4). To recover the optimal rate ( $h$ instead of $\left.h^{\frac{1}{2}}\right)$ it is necessary to construct $\psi_{h} \geq 0$ such that $\left(\frac{\partial u}{\partial \boldsymbol{n}}-\psi_{h}\right)$ decays like $h$ with respect to the norm of $H^{-\frac{1}{2}}\left(\Gamma_{C}\right)$, which is still an open question.

Working out the remaining term requires some preliminary technical steps. On account of $b\left(\psi_{h}, u_{h}\right) \geq 0$, and of the saturation condition, we derive

$$
\begin{aligned}
\int_{\Gamma_{C}} \psi_{h}\left(u-u_{h}\right) d \Gamma & \leq \int_{\Gamma_{C}} \psi_{h} u d \Gamma=\int_{\Gamma_{C}}\left(\psi_{h}-\frac{\partial u}{\partial \boldsymbol{n}}\right) u d \Gamma \\
& \leq \sum_{t \in \mathscr{T}_{h}^{C}} \int_{t}\left(\psi_{h}-\frac{\partial u}{\partial \boldsymbol{n}}\right) u d \Gamma \leq \sum_{t \in \mathscr{T}_{h}^{C}}\left\|\psi_{h}-\frac{\partial u}{\partial \boldsymbol{n}}\right\|_{L^{2}(t)}\|u\|_{L^{2}(t)} .
\end{aligned}
$$

Then, focussing on each segment $t$, if $u_{\mid T_{t}}>0$ then $\frac{\partial u}{\partial \boldsymbol{n}}{ }_{\mid T_{t}}=0$, and then, from Remark A.3 $\psi_{h \mid t}=0$ (see Appendix A for the notation $T_{t}$ ); otherwise, $u$ vanishes at least once in $T_{t}$. This results in, $\forall \boldsymbol{x} \in T_{t}$,

$$
|u(\boldsymbol{x})| \leq c h_{t}^{\frac{1}{2}}|u|_{H^{1}\left(T_{t}\right)},
$$

from which we deduce that

$$
\|u\|_{L^{2}(t)} \leq c h_{t}|u|_{H^{1}\left(T_{t}\right)} .
$$


Going back to (4.5) and using Cauchy-Schwartz inequality, we obtain

$$
\int_{\Gamma_{C}} \psi_{h}\left(u-u_{h}\right) d \Gamma \leq C \sum_{t \in \mathscr{T}_{h}^{C}} h_{t}^{\frac{1}{2}}\left\|\frac{\partial u}{\partial \boldsymbol{n}}\right\|_{H^{\frac{1}{2}\left(T_{t}\right)}} h_{t}|u|_{H^{1}\left(T_{t}\right)} \leq C h^{\frac{3}{2}}\|u\|_{H^{2}(\Omega)}^{2} .
$$

Putting together (4.4) and (4.6) finishes the proof of the lemma.

Proof of Theorem 4.3. Using Lemma 4.1 with the results of Lemmas 4.5 and 4.6 yields the inequality

$$
\left\|u-u_{h}\right\|_{H^{1}(\Omega)}^{2} \leq C\left(h^{\frac{1}{2}}\left\|u-u_{h}\right\|_{H^{1}(\Omega)}+h^{\frac{3}{2}}\|u\|_{H^{2}(\Omega)}\right)\|u\|_{H^{2}(\Omega)},
$$

from which we derive the final estimate (4.3).

Corollary 4.7. Let $(u, \varphi)$ be the solution of the mixed Signorini problem (2.13)(2.14), and $\left(u_{h}, \varphi_{h}\right) \in X_{h}(\Omega) \times M_{h}^{\varphi}\left(\Gamma_{C}\right)$ the solution of the discrete mixed inequality (3.2) $-\left(\right.$ (3.3) . Assume $u \in H^{2}(\Omega)$. Then

$$
\left\|\varphi-\varphi_{h}\right\|_{H^{-\frac{1}{2}}\left(\Gamma_{C}\right)} \leq C h^{\frac{1}{2}}\|u\|_{H^{2}(\Omega)} .
$$

Proof. Let $\psi_{h} \in M_{h}^{\varphi}\left(\Gamma_{C}\right)$ and $v_{h} \in X_{h}(\Omega)$. Then

$$
b\left(\varphi_{h}-\psi_{h}, v_{h}\right)=a\left(u_{h}, v_{h}\right)-L\left(v_{h}\right)-b\left(\psi_{h}, v_{h}\right)=a\left(u-u_{h}, v_{h}\right)+b\left(\varphi-\psi_{h}, v_{h}\right) .
$$

On account of the inf-sup condition of Lemma 3.1] and the triangle inequality,

$$
\left\|\varphi-\varphi_{h}\right\|_{H^{-\frac{1}{2}}\left(\Gamma_{C}\right)} \leq C\left(\left\|u-u_{h}\right\|_{H^{1}(\Omega)}+\inf _{\psi_{h} \in M_{h}^{\varphi}\left(\Gamma_{C}\right)}\left\|\varphi-\psi_{h}\right\|_{H^{-\frac{1}{2}}\left(\Gamma_{C}\right)}\right) .
$$

The estimate is completed by taking $\psi_{h}=r_{h}(\varphi) \in M_{h}^{\varphi}\left(\Gamma_{C}\right)$ and using Theorem 4.3

Remark 4.8. In the particular case where a Dirichlet boundary condition is applied to one (or both) of the extreme points of $\Gamma_{C}$-let us say $\boldsymbol{c}_{1}$, and recall that $t_{0} \in \mathscr{T}_{h}^{C}$ is the boundary element containing it - the trace operator maps $H_{0}^{1}\left(\Omega, \Gamma_{u}\right)$ onto $H_{00}^{\frac{1}{2}}\left(\Gamma_{C},\left\{\boldsymbol{c}_{1}\right\}\right)$ which coincides with the interpolated space $\left[H_{0}^{1}\left(\Gamma_{C},\left\{\boldsymbol{c}_{1}\right\}\right), L^{2}\left(\Gamma_{C}\right)\right]_{\frac{1}{2}}$ (see [27]). The construction of the Lagrange multipliers space $M_{h}^{\varphi}\left(\Gamma_{C}\right)$ requires some modifications. It is chosen to be a convex cone of the space

$$
\tilde{M}_{h}\left(\Gamma_{C}\right)=\left\{\psi_{h} \in W_{h}\left(\Gamma_{C}\right), \psi_{h \mid t_{0}} \in \mathcal{P}_{0}(t)\right\}
$$

involving all nonnegative functions

$$
M_{h}^{\varphi}\left(\Gamma_{C}\right)=\left\{\psi_{h} \in \tilde{M}_{h}\left(\Gamma_{C}\right), \psi_{h} \geq 0\right\} .
$$

The proof of the inf-sup condition on $b(.,$.$) restricted to \tilde{M}_{h}\left(\Gamma_{C}\right) \times X_{h}(\Omega)$ is based on the properties of the projection operator

$$
\tilde{\pi}_{h}: H_{00}^{\frac{1}{2}}\left(\Gamma_{C},\left\{\boldsymbol{c}_{1}\right\}\right) \rightarrow W_{h}^{0}\left(\Gamma_{C},\left\{\boldsymbol{c}_{1}\right\}\right)=\left\{\psi_{h} \in W_{h}\left(\Gamma_{C}\right), \psi_{h}\left(\boldsymbol{c}_{1}\right)=0\right\}
$$

defined as follows:

$$
\int_{\Gamma_{C}}\left(\varphi-\tilde{\pi}_{h} \varphi\right) \psi_{h} d \Gamma=0, \quad \forall \psi_{h} \in \tilde{M}_{h}\left(\Gamma_{C}\right) .
$$

Here again under the Crouzeix-Thomée criterion (3.4) on the mesh $\mathscr{T}_{h}^{C}$ we have the uniform stability (see [4], Lemma 2.2 , or [30])

$$
\left\|\tilde{\pi}_{h} \psi\right\|_{H_{00}^{\frac{1}{2}}\left(\Gamma_{C},\left\{\boldsymbol{c}_{1}\right\}\right)} \leq C\|\psi\|_{\left.H_{00}^{\frac{1}{2}}\left(\Gamma_{C},\left\{\boldsymbol{c}_{1}\right\}\right)\right)} .
$$


This operator is called the mortar projector, and is used to enforce weak continuity conditions of finite element functions at the interfaces of nonmatching grids (see [9], [7], [2]). As a consequence, the inf-sup condition given in Lemma 3.1 is still valid when the norm of $H^{-\frac{1}{2}}\left(\Gamma_{C}\right)$ is replaced by the more appropriate norm of $H_{00}^{\frac{1}{2}}\left(\Gamma_{C},\left\{\boldsymbol{c}_{1}\right\}\right)^{\prime}$. As a corollary, the well posedness for the discrete mixed problem of Proposition 3.2 holds true, and the approximation results of Theorem 4.3 and Corollary 4.7 are also valid in the sense that if $u \in H^{2}(\Omega)$, then

$$
\left\|u-u_{h}\right\|_{H^{1}(\Omega)}+\left\|\varphi-\varphi_{h}\right\|_{H_{00}^{\frac{1}{2}}\left(\Gamma_{C},\left\{\boldsymbol{c}_{1}\right\}\right)^{\prime}} \leq C h^{\frac{1}{2}}\|u\|_{H^{2}(\Omega)} .
$$

\section{Mixed Finite Elements AND CONFORMing DISCRETIZATION OF PROBLEM (2.9)}

Enforcing explicitly $u_{h \mid \Gamma_{C}} \geq 0$ requires that we use the polar convex cone of $M_{h}^{\varphi}\left(\Gamma_{C}\right)$, denoted $M_{h}^{u}\left(\Gamma_{C}\right)$ and characterized as the subset of $W_{h}\left(\Gamma_{C}\right)$ containing all "weakly" nonnegative functions,

$$
M_{h}^{u}\left(\Gamma_{C}\right)=\left\{\psi_{h} \in W_{h}\left(\Gamma_{C}\right), \quad \int_{\Gamma_{C}} \psi_{h} \chi_{h} d \Gamma \geq 0, \quad \forall \chi_{h} \in M_{h}^{\varphi}\left(\Gamma_{C}\right)\right\} .
$$

If $\left(u_{h}, \varphi_{h}\right)$ is the solution to the discrete mixed finite element problem (3.2)-(3.3) set on $X_{h}(\Omega) \times M_{h}^{u}\left(\Gamma_{C}\right)$, then $u_{h}$ belongs to

$$
K_{h}^{u}(\Omega)=\left\{v_{h} \in X_{h}(\Omega), b\left(\psi_{h}, v_{h}\right) \geq 0, \forall \psi_{h} \in M_{h}^{u}\left(\Gamma_{C}\right)\right\} .
$$

By the Hahn-Banach theorem we have explicitly

$$
K_{h}^{u}(\Omega)=\left\{v_{h} \in X_{h}(\Omega), v_{h \mid \Gamma_{C}} \geq 0\right\} .
$$

Besides, $u_{h}$ is the unique solution of the discrete Signorini variational inequality: find $u_{h} \in K_{h}^{u}(\Omega)$ such that

$$
\text { (4.1) is satisfied, } \quad \forall v_{h} \in K_{h}^{u}(\Omega) \text {. }
$$

The convex set $K_{h}^{u}(\Omega)$ is an internal approximation of $K(\Omega)$, i.e., $K_{h}(\Omega) \subset K(\Omega)$; the discretization of the Signorini problem (2.9) is then conforming in the Hodge sense.

The numerical analysis of this problem starts by deriving an error estimate for $u$, and then we need another adaptation of Falk's results to our conforming case.

Lemma 5.1. Let $u \in K(\Omega)$ be the solution to the variational Signorini inequality (2.9), and $u_{h} \in K_{h}^{u}(\Omega)$ the solution to the discrete variational inequality (5.1).

Then

$$
\left\|u-u_{h}\right\|_{H^{1}(\Omega)}^{2} \leq C \inf _{v_{h} \in K_{h}^{u}(\Omega)}\left(\left\|u-v_{h}\right\|_{H^{1}(\Omega)}^{2}+\left\langle\frac{\partial u}{\partial \boldsymbol{n}}, v_{h}\right\rangle_{\frac{1}{2}, \partial \Omega}-\left\langle g, v_{h}\right\rangle_{\frac{1}{2}, \Gamma_{g}}\right) .
$$

Proof. It is direct from Lemma 4.1, where the consistency error is canceled because the approximation is conforming.

Theorem 5.2. Let $u \in K(\Omega)$ be the solution to the continuous variational Signorini problem (2.9), and $u_{h} \in K_{h}^{u}(\Omega)$ the "conforming" piecewise linear approximation of $u$, the solution to (5.1). Assume $u \in H^{2}(\Omega)$. Then

$$
\left\|u-u_{h}\right\|_{H^{1}(\Omega)} \leq C h^{\frac{3}{4}}\|u\|_{H^{2}(\Omega)} .
$$


Moreover, if in addition the number of points in $\Gamma_{C}$ where the constraint changes from binding to nonbinding is finite then

$$
\left\|u-u_{h}\right\|_{H^{1}(\Omega)} \leq C h|\log (h)|^{\frac{1}{4}}\|u\|_{H^{2}(\Omega)} .
$$

Proof. The proof is based on the choice $v_{h}=\mathcal{I}_{h} u \in K_{h}^{u}(\Omega)$ (5.2) is shown in a standard way (see [22], for instance). The estimate (5.3) is slightly better than the convergence rate of order $h|\log (h)|^{\frac{1}{2}}$, given in [3] , and is proven as follows.

Consider $\Gamma_{B}$ and $\Gamma_{N}$, the portions of $\Gamma_{C}$ defined respectively by $u_{\mid \Gamma_{B}}=0$ and $u_{\mid \Gamma_{N}}>0$; therefore $\left(\frac{\partial u}{\partial \boldsymbol{n}}\right)_{\mid \Gamma_{N}}=0$. Then, select, among the triangles $\kappa \in \mathscr{T}_{h}$ having a complete edge contained in $\Gamma_{C}$, those for which $\bar{\kappa} \cap \Gamma_{B} \neq \emptyset$ and $\bar{\kappa} \cap \Gamma_{N} \neq \emptyset$. Their number is bounded uniformly in $h$ in view of the additional assumption; the set of such elements is denoted $s_{h}$. Looking at the product $\left(\frac{\partial u}{\partial \boldsymbol{n}}\left(\mathcal{I}_{h} u\right)\right)_{\mid \kappa \cap \Gamma_{C}}$, we see that because of the saturation condition it vanishes except for $\kappa \in s_{h}$. Thus,

$$
\int_{\Gamma_{C}} \frac{\partial u}{\partial \boldsymbol{n}}\left(\mathcal{I}_{h} u\right) d \Gamma=\sum_{\kappa \in s_{h}} \int_{\bar{\kappa} \cap \Gamma_{C}} \frac{\partial u}{\partial \boldsymbol{n}}\left(\mathcal{I}_{h} u\right) d \Gamma=\sum_{\kappa \in s_{h}} \int_{\bar{\kappa} \cap \Gamma_{C}} \frac{\partial u}{\partial \boldsymbol{n}}\left(\mathcal{I}_{h} u-u\right) d \Gamma .
$$

For any real number $p$ in $] 1,2]$ and $p^{\prime} \in\left[2,+\infty\right.$ [its conjugate number, i.e., $\frac{1}{p}+\frac{1}{p}^{\prime}=1$, the Hölder inequality produces

$$
\begin{aligned}
\int_{\Gamma_{C}} \frac{\partial u}{\partial \boldsymbol{n}}\left(\mathcal{I}_{h} u\right) d \Gamma & \leq \sum_{\kappa \in s_{h}}\left\|\frac{\partial u}{\partial \boldsymbol{n}}\right\|_{L^{p^{\prime}\left(\kappa \cap \Gamma_{C}\right)}}\left\|\mathcal{I}_{h} u-u\right\|_{L^{p}\left(\bar{\kappa} \cap \Gamma_{C}\right)} \\
& \leq \sum_{\kappa \in s_{h}}\left\|\frac{\partial u}{\partial \boldsymbol{n}}\right\|_{L^{p^{\prime}\left(\kappa \cap \Gamma_{C}\right)}} h^{\frac{1}{p}}\left\|\mathcal{I}_{h} u-u\right\|_{L^{\infty}\left(\bar{\kappa} \cap \Gamma_{C}\right)} .
\end{aligned}
$$

Having in mind that the cardinality of the set $s_{h}$ is bounded uniformly in $h$, we get

$$
\left|\int_{\Gamma_{C}} \frac{\partial u}{\partial \boldsymbol{n}}\left(\mathcal{I}_{h} u\right) d \Gamma\right| \leq C h^{\frac{1}{p}}\left\|\frac{\partial u}{\partial \boldsymbol{n}}\right\|_{L^{p^{\prime}\left(\Gamma_{C}\right)}}\left\|\mathcal{I}_{h} u-u\right\|_{L^{\infty}\left(\Gamma_{C}\right)} .
$$

In one side, using the Sobolev-Morrey theorem, we obtain

$$
\left\|\frac{\partial u}{\partial \boldsymbol{n}}\right\|_{L^{p^{\prime}}\left(\Gamma_{C}\right)} \leq C \sqrt{p^{\prime}}\left\|\frac{\partial u}{\partial \boldsymbol{n}}\right\|_{H^{\frac{1}{2}\left(\Gamma_{C}\right)}} \leq C \sqrt{p^{\prime}}\|u\|_{H^{2}(\Omega)},
$$

with $C$ independent of $p^{\prime}$. In the other side the Gagliardo-Nirenberg inequality implies that

$$
\left\|\mathcal{I}_{h} u-u\right\|_{L^{\infty}\left(\Gamma_{C}\right)} \leq C\left\|\mathcal{I}_{h} u-u\right\|_{L^{2}\left(\Gamma_{C}\right)}^{\frac{1}{2}}\left\|\mathcal{I}_{h} u-u\right\|_{H^{1}\left(\Gamma_{C}\right)}^{\frac{1}{2}} \leq C h\|u\|_{H^{2}(\Omega)} .
$$

Inserting these in (3.4) leads to

$$
\int_{\Gamma_{C}} \frac{\partial u}{\partial \boldsymbol{n}}\left(\mathcal{I}_{h} u\right) d \Gamma \leq C h^{2}\left(h^{-\frac{1}{p}^{\prime}} \sqrt{p^{\prime}}\right)\|u\|_{H^{2}(\Omega)}^{2} .
$$

Taking $p^{\prime}=|\log h|$ finishes the proof.

Remark 5.3. Although the additional assumption in Theorem 5.2 (that is, the number of points where the constraints $\frac{\partial u}{\partial \boldsymbol{n}}$ become effective is finite) is most often valid, it seems to be a working hypothesis currently used and has appeared first in [11]. In particular, from the solid mechanics point of view, it appears to be a fair modeling of realistic unilateral situations which engineers are interested in. Conversely, if this hypothesis is violated, it is not possible to find out, through the numerical results, that the points where the type of contact condition is changed, 
are of infinite number. This might be a justification of the loss of optimality, in this case.

Corollary 5.4. Let $(u, \varphi)$ be the solution to the mixed Signorini problem (2.13)(2.14), and $\left(u_{h}, \varphi_{h}\right) \in X_{h}(\Omega) \times M_{h}^{u}\left(\Gamma_{C}\right)$ the solution to the discrete mixed inequality (3.2) -(3.3). Assume $u \in H^{2}(\Omega)$. Then

$$
\left\|\varphi-\varphi_{h}\right\|_{H^{-\frac{1}{2}}\left(\Gamma_{C}\right)} \leq C h^{\frac{3}{4}}\|u\|_{H^{2}(\Omega)} .
$$

Moreover, if in addition the number of points in $\Gamma_{C}$ where the constraint changes from binding to nonbinding is finite, then

$$
\left\|\varphi-\varphi_{h}\right\|_{H^{-\frac{1}{2}}\left(\Gamma_{C}\right)} \leq C h|\log (h)|^{\frac{1}{4}}\|u\|_{H^{2}(\Omega)} .
$$

Proof. Proceeding as in the proof of Corollary 4.7, we get

$$
\left\|\varphi-\varphi_{h}\right\|_{H^{-\frac{1}{2}}\left(\Gamma_{C}\right)} \leq C\left(\left\|u-u_{h}\right\|_{H^{1}(\Omega)}+\inf _{\psi_{h} \in M_{h}^{u}\left(\Gamma_{C}\right)}\left\|\varphi-\psi_{h}\right\|_{H^{-\frac{1}{2}}\left(\Gamma_{C}\right)}\right) .
$$

Finally, choose $\psi_{h}=\pi_{h} \varphi$ which belongs to $M_{h}^{u}\left(\Gamma_{C}\right)$, and use Theorem 5.2 and the estimate (3.6).

Remark 5.5. Handling the particular situation described in Remark 4.8, where a Dirichlet boundary condition is applied to one or two extreme points of $\Gamma_{C}$ (say $\boldsymbol{c}_{1}$ ), also requires a change in the construction of the Lagrange multipliers space $M_{h}^{u}\left(\Gamma_{C}\right)$. It is defined to be the convex cone

$$
M_{h}^{u}\left(\Gamma_{C}\right)=\left\{\psi_{h} \in \tilde{M}_{h}\left(\Gamma_{C}\right), \quad \int_{\Gamma_{C}} \psi_{h} \chi_{h} \geq 0, \quad \forall \chi_{h} \in W_{h}^{0}\left(\Gamma_{C},\left\{\boldsymbol{c}_{1}\right\}\right), \chi_{h} \geq 0\right\} .
$$

Identifying $\tilde{M}_{h}\left(\Gamma_{C}\right)$ to the dual space of $W_{h}^{0}\left(\Gamma_{C},\left\{\boldsymbol{c}_{1}\right\}\right)$ and using the Hahn-Banach Theorem again, we get

$$
K_{h}^{u}(\Omega)=\left\{v_{h} \in X_{h}(\Omega), v_{h \mid \Gamma_{C}} \geq 0\right\} .
$$

The error estimate on $u$ is carried out exactly as in Theorem 5.2, and the convergence results given there still hold true. However, studying the accuracy of the method on $\varphi$ is based on an optimal approximation estimate, in the $H_{00}^{\frac{1}{2}}\left(\Gamma_{C},\left\{\boldsymbol{c}_{1}\right\}\right)^{\prime}$ norm, of some projection operator $\check{\pi}_{h}: L^{2}\left(\Gamma_{C}\right) \rightarrow W_{h}\left(\Gamma_{C}\right)$ constructed and analyzed in Appendix B. Consequently, choosing $\psi_{h}=\check{\pi}_{h} \varphi$ in (5.5) in view of (B.2) yields the same bound as Corollary 5.4 under the same hypothesis.

\section{Bubble-stabilized mixed finite Elements}

Many authors (see [26], for instance) use piecewise constant Lagrange multipliers, meaning that they work with the space

$$
N_{h}\left(\Gamma_{C}\right)=\left\{\psi_{h} \in L^{2}\left(\Gamma_{C}\right), \quad \forall t \in \mathscr{T}_{h}^{C}, \psi_{h \mid t} \in \mathcal{P}_{0}(t), \psi_{h} \geq 0\right\} .
$$

The numerical analysis of the resulting mixed problem set on $X_{h}(\Omega) \times N_{h}\left(\Gamma_{C}\right)$ allows one to exhibit a satisfactory convergence rate. Actually, if $u \in H^{2}(\Omega)$, it is proven in [26] that

$$
\left\|u-u_{h}\right\|_{H^{1}(\Omega)}+\left\|\varphi-\varphi_{h}\right\|_{H^{-\frac{1}{2}}\left(\Gamma_{C}\right)} \leq C h^{\frac{3}{4}}\|u\|_{H^{2}(\Omega)} .
$$

Unfortunately this mixed finite element discretization has an essential drawback. It works only in the particular situation where neither of the extreme points of $\Gamma_{C}$ 


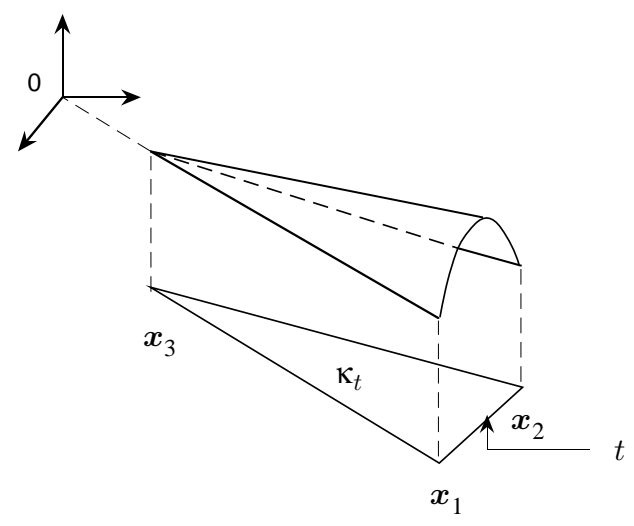

FiguRe 6.1.

is subjected to a Dirichlet condition. Otherwise, the space $N_{h}\left(\Gamma_{C}\right)$ contains one (if only one point of $\left\{\boldsymbol{c}_{1}, \boldsymbol{c}_{2}\right\}$ is subjected to a Dirichlet boundary condition) or two (if both extreme points of $\Gamma_{C}$ are subjected to Dirichlet conditions) spurious modes, and the well posedness of the discrete equations (3.2)-(3.3) is definitely lost. Besides, there is no hope that it could be extended to three-dimensional problems, as it is well known that the number of triangles in $\Gamma_{C}$ is drastically higher than the number of the degrees of freedom attached to $\Gamma_{C}$. This results necessarily in a great number of spurious modes, and the discrete problem is ill-posed.

A possible way to avoid the difficulties of this approach is to stabilize the approximation by introducing the bubble finite element functions. This approach is inspired by the work of [10] and [12], where it is used in some nonconforming domain decomposition algorithms and proved to perform well. The discrete space will be enriched by some bubble functions defined on each $t \in \mathscr{T}_{h}^{C}$. Precisely, let there be given an element $t \in \mathscr{T}_{h}^{C}$ with vertices $\left\{\boldsymbol{x}_{1}, \boldsymbol{x}_{2}\right\}$ and $\kappa=\kappa_{t}$ the (unique!) triangle of $\mathscr{T}_{h}$ having $t$ as an edge. The vertices of $\kappa$ are $\left(\boldsymbol{x}_{i}\right)_{1 \leq i \leq 3}$, and $\lambda_{i},(1 \leq i \leq 3)$ is the barycentric coordinate associated with $\boldsymbol{x}_{i}$. The bubble function we need to use is defined to be (see Figure 6.1 for its shape)

$$
\varphi_{t}(\boldsymbol{x})=\frac{6}{|t|} \lambda_{1}(\boldsymbol{x}) \lambda_{2}(\boldsymbol{x}), \quad \forall \boldsymbol{x} \in \kappa,
$$

and extended by zero elsewhere. The normalization coefficient allows us to have $\int_{t} \varphi_{t} d \Gamma=1$.

The function $\varphi_{t}$ is continuous and vanishes on the skeleton of the mesh, except on $t$, where it is explicitly given by

$$
\left(\varphi_{t}\right)_{\mid t}(\boldsymbol{x})=\frac{6}{|t|^{3}}\left(\boldsymbol{x}-\boldsymbol{x}_{i}, \boldsymbol{x}_{i+1}-\boldsymbol{x}\right)_{\mathbb{R}^{2}} .
$$

Then, the finite element space, where $u_{h}$ is computed, is given by

$$
Y_{h}(\Omega)=X_{h}(\Omega) \oplus\left(\bigoplus_{t \in \mathscr{T}_{h}^{C}} \mathbb{R} \varphi_{t}\right) .
$$

Setting as degrees of freedom

$$
\Sigma=\left\{v_{h}(\boldsymbol{x}), \boldsymbol{x} \in \Xi_{h}, \int_{t}\left(v_{h}-\mathcal{I}_{h} v_{h}\right) d \Gamma, t \in \mathscr{T}_{h}^{C}\right\}
$$


it is not hard to show that $\left(\Omega, Y_{h}(\Omega), \Sigma\right)$ is unisolvent. We also need to introduce the interpolation operator $\mathcal{J}_{h}$, defined for any $v \in \mathscr{C}(\bar{\Omega})$ as follows:

$$
\mathcal{J}_{h} v=\mathcal{I}_{h} v+\sum_{t \in \mathscr{T}_{h}^{C}}\left(\int_{t}\left(v-\mathcal{I}_{h} v\right) d \Gamma\right) \varphi_{t}
$$

The operator $\mathcal{J}_{h}$ leaves the elements of $X_{h}(\Omega)$ invariant, and as a consequence, for any $(\mu, \nu) \in[0,1] \times] 1,2]$, the following error estimate holds: $\forall v \in H^{\nu}(\Omega)$,

$$
\left\|v-\mathcal{J}_{h} v\right\|_{H^{\mu}(\Omega)} \leq C h^{\nu-\mu}\|v\|_{H^{\nu}(\Omega)}
$$

We are now in position to pose the discrete stabilized mixed finite element problem: find $\left(u_{h}, \varphi_{h}\right) \in Y_{h}(\Omega) \times N_{h}\left(\Gamma_{C}\right)$ such that

$$
\begin{aligned}
a\left(u_{h}, v_{h}\right)-b\left(\varphi_{h}, v_{h}\right) & =L\left(v_{h}\right), \quad \forall v_{h} \in Y_{h}(\Omega), \\
b\left(\psi_{h}-\varphi_{h}, u_{h}\right) & \geq 0, \quad \forall \psi_{h} \in N_{h}\left(\Gamma_{C}\right) .
\end{aligned}
$$

Remark 6.1. The range of the space $Y_{h}(\Omega)$ under the trace operator on $\Gamma_{C}$ is the quadratic Lagrangian finite element space

$$
V_{h}\left(\Gamma_{C}\right)=\left\{\chi_{h} \in \mathscr{C}\left(\bar{\Gamma}_{C}\right), \quad \forall t \in \mathscr{T}_{h}^{C}, \chi_{h \mid t} \in \mathcal{P}_{2}(t)\right\} .
$$

The idea of approximating $u_{\mid \Gamma_{C}}$ by quadratic finite elements and the flux $\varphi=\frac{\partial u}{\partial \boldsymbol{n}}$ by a piecewise constant function is not new; it was also used in the $\mathcal{P}_{2} \times \mathcal{P}_{0}$ mixed finite elements discretization of Poisson problem (see [29], [32]).

Remark 6.2. It is sufficient for the stabilization to add the bubble functions only to the external elements $t_{0}$ and $t_{i^{*}-1}$ of $\Gamma_{C}$, those that contain $\boldsymbol{c}_{1}$ or $\boldsymbol{c}_{2}$. The analysis is carried out following the same arguments as presented below.

The proof of the well posedness of the mixed variational inequality relies on a uniform inf-sup condition which is provided by

Lemma 6.3. Under the Crouzeix-Thomée assumption (3.4) on the mesh $\mathscr{T}_{h}^{C}$, the following inf-sup condition holds:

$$
\inf _{\psi_{h} \in N_{h}\left(\Gamma_{C}\right)} \sup _{v_{h} \in Y_{h}(\Omega)} \frac{b\left(\psi_{h}, v_{h}\right)}{\left\|v_{h}\right\|_{H^{1}(\Omega)}\left\|\psi_{h}\right\|_{H^{-\frac{1}{2}}\left(\Gamma_{C}\right)}} \geq \gamma^{\prime} .
$$

The constant $\gamma^{\prime}>0$ and does not depend on $h$.

Proof. We follow the same lines as for Lemma 3.1 For any $\psi_{h} \in N_{h}\left(\Gamma_{C}\right)$ we intend to construct $v_{h} \in Y_{h}(\Omega)$ satisfying (3.7).

Let $v \in H^{1}(\Omega)$ be the solution to the Laplace problem (3.8). By a stable finite element extension as in [8], it is possible to construct $v_{h} \in Y_{h}(\Omega)$ such that

$$
v_{h \mid \Gamma_{C}}=\tilde{r}_{h} v_{\mid \Gamma_{C}}+\sum_{t \in \mathscr{T}_{h}^{C}}\left(\int_{t}\left(v-\tilde{r}_{h} v\right) d \Gamma\right) \varphi_{t} \quad \text { and } \quad\left\|v_{h}\right\|_{H^{1}(\Omega)} \leq c\left\|v_{h}\right\|_{H^{\frac{1}{2}}\left(\Gamma_{C}\right)},
$$

$\tilde{r}_{h}$ being the standard Clément regularizing operator defined in Appendix A. The constant $c$ is independent of $h$. Then, computing $b\left(\psi_{h}, v_{h}\right)$ and using (3.11) yields

$$
b\left(\psi_{h}, v_{h}\right)=\sum_{t \in \mathscr{T}_{h}^{C}} \psi_{h \mid t} \int_{t} v d \Gamma=\int_{\Gamma_{C}} \psi_{h} v d \Gamma=b\left(\psi_{h}, v\right) \geq c\left\|\psi_{h}\right\|_{H^{-\frac{1}{2}}\left(\Gamma_{C}\right)}^{2} .
$$

In order to show that

$$
\left\|v_{h}\right\|_{H^{\frac{1}{2}\left(\Gamma_{C}\right)}} \leq c\|v\|_{H^{\frac{1}{2}}\left(\Gamma_{C}\right)} \leq c\left\|\psi_{h}\right\|_{H^{-\frac{1}{2}}\left(\Gamma_{C}\right)},
$$


we first need to bound

$$
\begin{aligned}
\left\|\sum_{t \in \mathscr{T}_{h}^{C}}\left(\int_{t}\left(v-\tilde{r}_{h} v\right) d \Gamma\right) \varphi_{t}\right\|_{L^{2}\left(\Gamma_{C}\right)}^{2} & =\sum_{t \in \mathscr{T}_{h}^{C}}\left(\int_{t}\left(v-\tilde{r}_{h} v\right) d \Gamma\right)^{2}\left\|\varphi_{t}\right\|_{L^{2}(t)}^{2} \\
& \leq \frac{6}{5} \sum_{t \in \mathscr{T}_{h}^{C}}|t|^{-1}\left(\int_{t}\left(v-\tilde{r}_{h} v\right) d \Gamma\right)^{2} \\
& \leq \frac{6}{5} \sum_{t \in \mathscr{T}_{h}^{C}}\left\|v-\tilde{r}_{h} v\right\|_{L^{2}(t)}^{2} .
\end{aligned}
$$

Using (A.4) with $\nu=0$ produces

$$
\left\|\sum_{t \in \mathscr{T}_{h}^{C}}\left(\int_{t}\left(v-\tilde{r}_{h} v\right) d \Gamma\right) \varphi_{t}\right\|_{L^{2}\left(\Gamma_{C}\right)}^{2} \leq c \sum_{t \in \mathscr{T}_{h}^{C}}\|v\|_{L^{2}\left(T_{t}\right)}^{2} \leq c\|v\|_{L^{2}\left(\Gamma_{C}\right)}^{2} .
$$

In the other side,

$$
\begin{aligned}
\left|\sum_{t \in \mathscr{T}_{h}^{C}}\left(\int_{t}\left(v-\tilde{r}_{h} v\right) d \Gamma\right) \varphi_{t}\right|_{H^{1}\left(\Gamma_{C}\right)}^{2} & =\sum_{t \in \mathscr{T}_{h}^{C}}\left(\int_{t}\left(v-\tilde{r}_{h} v\right) d \Gamma\right)^{2}\left|\varphi_{t}\right|_{H^{1}(t)}^{2} \\
& \leq 12 \sum_{t \in \mathscr{T}_{h}^{C}}|t|^{-3}\left(\int_{t}\left(v-\tilde{r}_{h} v\right) d \Gamma\right)^{2} \\
& \leq 12 \sum_{t \in \mathscr{T}_{h}^{C}}|t|^{-2}\left\|v-\tilde{r}_{h} v\right\|_{L^{2}(t)}^{2} .
\end{aligned}
$$

By A.3 we have

$$
\left|\sum_{t \in \mathscr{T}_{h}^{C}}\left(\int_{t}\left(v-\tilde{r}_{h} v\right) d \Gamma\right) \varphi_{t}\right|_{H^{1}\left(\Gamma_{C}\right)}^{2} \leq c \sum_{t \in \mathscr{T}_{h}^{C}}|v|_{H^{1}\left(T_{t}\right)}^{2} \leq c|v|_{H^{1}\left(\Gamma_{C}\right)}^{2} .
$$

Finally, again on account of (A.4) with $\nu=0$ and $\nu=1$, we obtain

$$
\left\|v_{h}\right\|_{L^{2}\left(\Gamma_{C}\right)} \leq c\|v\|_{L^{2}\left(\Gamma_{C}\right)} \quad \text { and } \quad\left\|v_{h}\right\|_{H^{1}\left(\Gamma_{C}\right)} \leq c\|v\|_{H^{1}\left(\Gamma_{C}\right)} .
$$

The stability (6.4) is derived by Hilbert interpolation.

Proposition 6.4. The discrete mixed finite element problem (6.2)-(6.3) has only one solution in $Y_{h}(\Omega) \times N_{h}\left(\Gamma_{C}\right)$ such that

$$
\left\|u_{h}\right\|_{H^{1}(\Omega)}+\left\|\varphi_{h}\right\|_{H^{-\frac{1}{2}\left(\Gamma_{C}\right)}} \leq C\left(\|f\|_{L^{2}(\Omega)}+\|g\|_{H^{-\frac{1}{2}}\left(\Gamma_{g}\right)}\right) .
$$

The constant $C$ does not depend on $h$.

As in the previous sections, we begin the numerical analysis by proving an error estimate for $u$. Then, we introduce the following convex cone:

$$
\begin{aligned}
K_{h}(\Omega) & =\left\{v_{h} \in Y_{h}(\Omega), b\left(\psi_{h}, v_{h}\right) \geq 0, \forall \psi_{h} \in N_{h}\left(\Gamma_{C}\right)\right\} \\
& =\left\{v_{h} \in Y_{h}(\Omega), \quad \int_{t} v_{h} d \Gamma \geq 0\right\} .
\end{aligned}
$$

The function $u_{h}$ belongs to $K_{h}(\Omega)$ and is the unique solution to the discrete Signorini problem: find $u_{h} \in K_{h}(\Omega)$ such that

$$
\text { (4.1) is satisfied, } \forall v_{h} \in K_{h}(\Omega) \text {. }
$$


As we have $K_{h}(\Omega) \not \subset K(\Omega), K_{h}(\Omega)$ is then an external approximation $K(\Omega)$. The discretization of the formulation (2.9) of Signorini's problem by the finite element variational inequality (6.5) is nonconforming. Nevertheless, the convergence rate of this algorithm is "quasi-optimal".

Theorem 6.5. Let $u \in K(\Omega)$ be the solution to the continuous variational Signorini problem (2.9), and $u_{h} \in K_{h}(\Omega)$ the "nonconforming" stabilized linear finite element approximation of $u$, the solution to (6.5). Assume $u \in H^{2}(\Omega)$. Then

$$
\left\|u-u_{h}\right\|_{H^{1}(\Omega)} \leq C h^{\frac{3}{4}}\|u\|_{H^{2}(\Omega)} .
$$

Moreover, if in addition the number of points in $\Gamma_{C}$ where the constraint changes from binding to nonbinding is finite, then

$$
\left\|u-u_{h}\right\|_{H^{1}(\Omega)} \leq C h|\log (h)|^{\frac{1}{4}}\|u\|_{H^{2}(\Omega)} .
$$

The proof is based on Lemma 4.1 and needs two intermediary results. The first is the bound for the approximation error.

Lemma 6.6. Let $u \in K(\Omega)$ be the solution of the continuous Signorini problem (2.9), and $u_{h} \in K_{h}(\Omega)$ the solution to (6.5). Assume $u \in H^{2}(\Omega)$. Then

$$
\inf _{v_{h} \in K_{h}(\Omega)}\left(\left\|u-v_{h}\right\|_{H^{1}(\Omega)}^{2}+\int_{\Gamma_{C}} \frac{\partial u}{\partial \boldsymbol{n}} v_{h} d \Gamma\right) \leq C h^{2}\|u\|_{H^{2}(\Omega)}^{2} .
$$

Proof. Choose $v_{h}=\mathcal{J}_{h} u$. It is easily checked that $\forall \psi_{h} \in N_{h}\left(\Gamma_{C}\right)$,

$$
b\left(\psi_{h}, v_{h}\right)=b\left(\psi_{h}, u\right)=\int_{\Gamma_{C}} \psi_{h} u d \Gamma \geq 0 .
$$

Then $v_{h} \in K_{h}(\Omega)$. Bounding $\left\|u-v_{h}\right\|_{H^{1}(\Omega)}$ is direct from (6.1). The integral term is treated in the following way, using the saturation condition:

$$
\int_{\Gamma_{C}} \frac{\partial u}{\partial \boldsymbol{n}} v_{h} d \Gamma=\int_{\Gamma_{C}} \frac{\partial u}{\partial \boldsymbol{n}}\left(\mathcal{J}_{h} u-u\right) d \Gamma .
$$

The properties of $\mathcal{J}_{h}$ allow to write, $\forall \psi_{h} \in N_{h}\left(\Gamma_{C}\right)$,

$\int_{\Gamma_{C}} \frac{\partial u}{\partial \boldsymbol{n}} v_{h} d \Gamma=\int_{\Gamma_{C}}\left(\frac{\partial u}{\partial \boldsymbol{n}}-\psi_{h}\right)\left(\mathcal{J}_{h} u-u\right) d \Gamma \leq\left\|\frac{\partial u}{\partial \boldsymbol{n}}-\psi_{h}\right\|_{L^{2}\left(\Gamma_{C}\right)}\left\|\mathcal{J}_{h} u-u\right\|_{L^{2}\left(\Gamma_{C}\right)}$.

We conclude by using the standard approximation.

Lemma 6.7. Let $u \in K(\Omega)$ be the solution to the continuous Signorini problem (2.9), and $u_{h} \in K_{h}(\Omega)$ the solution to (6.5). Assume $u \in H^{2}(\Omega)$. Then the consistency error is bounded as follows:

$$
\inf _{v \in K(\Omega)} \int_{\Gamma_{C}} \frac{\partial u}{\partial \boldsymbol{n}}\left(v-u_{h}\right) d \Gamma \leq C\left(h^{\frac{3}{2}}\|u\|_{H^{2}(\Omega)}+h\left\|u-u_{h}\right\|_{H^{1}(\Omega)}\right)\|u\|_{H^{2}(\Omega)} .
$$

Moreover, if in addition the number of points in $\Gamma_{C}$ where the constraint changes from binding to nonbinding is finite, then

$$
\inf _{v \in K(\Omega)} \int_{\Gamma_{C}} \frac{\partial u}{\partial \boldsymbol{n}}\left(v-u_{h}\right) d \Gamma \leq C\left(h^{2}|\log h|^{\frac{1}{2}}\|u\|_{H^{2}(\Omega)}+h\left\|u-u_{h}\right\|_{H^{1}(\Omega)}\right)\|u\|_{H^{2}(\Omega)} .
$$


Proof. It is similar to that of Lemma 4.6. Choose $v=u$; then we have, $\forall \psi_{h} \in$ $N_{h}\left(\Gamma_{C}\right)$,

$$
\int_{\Gamma_{C}} \frac{\partial u}{\partial \boldsymbol{n}}\left(u-u_{h}\right) d \Gamma=\int_{\Gamma_{C}}\left(\frac{\partial u}{\partial \boldsymbol{n}}-\psi_{h}\right)\left(u-u_{h}\right) d \Gamma+\int_{\Gamma_{C}} \psi_{h}\left(u-u_{h}\right) d \Gamma .
$$

The first part is handled as follows:

$$
\int_{\Gamma_{C}}\left(\frac{\partial u}{\partial \boldsymbol{n}}-\psi_{h}\right)\left(u-u_{h}\right) d \Gamma \leq\left\|\frac{\partial u}{\partial \boldsymbol{n}}-\psi_{h}\right\|_{H^{-\frac{1}{2}\left(\Gamma_{C}\right)}}\left\|u-u_{h}\right\|_{H^{\frac{1}{2}}\left(\Gamma_{C}\right)} .
$$

If we take $\psi_{h \mid t}=|t|^{-1} \int_{t} \frac{\partial u}{\partial \boldsymbol{n}} d \Gamma \geq 0$, then $\psi_{h} \in N_{h}\left(\Gamma_{C}\right)$; it is actually the orthogonal projection of $\frac{\partial u}{\partial \boldsymbol{n}}$ on piecewise constant functions. By duality we obtain

$$
\left\|\frac{\partial u}{\partial \boldsymbol{n}}-\psi_{h}\right\|_{H^{-\frac{1}{2}\left(\Gamma_{C}\right)}} \leq \operatorname{ch}\|u\|_{H^{2}(\Omega)} .
$$

Therefore

$$
\int_{\Gamma_{C}}\left(\frac{\partial u}{\partial \boldsymbol{n}}-\psi_{h}\right)\left(u-u_{h}\right) d \Gamma \leq c h\|u\|_{H^{2}(\Omega)}\left\|u-u_{h}\right\|_{H^{\frac{1}{2}}\left(\Gamma_{C}\right)} .
$$

Next, focussing on the remaining part, observe that, due to the unilateral condition,

$$
\int_{\Gamma_{C}} \psi_{h}\left(u-u_{h}\right) d \Gamma \leq \int_{\Gamma_{C}} \psi_{h} u d \Gamma .
$$

By the saturation we have

$$
\int_{\Gamma_{C}} \psi_{h} u d \Gamma=\int_{\Gamma_{C}}\left(\psi_{h}-\frac{\partial u}{\partial \boldsymbol{n}}\right) u d \Gamma .
$$

Or equivalently, $\forall \chi_{h} \in N_{h}\left(\Gamma_{C}\right)$,

$$
\int_{\Gamma_{C}} \psi_{h} u d \Gamma=\int_{\Gamma_{C}}\left(\psi_{h}-\frac{\partial u}{\partial \boldsymbol{n}}\right)\left(u-\chi_{h}\right) d \Gamma
$$

Choosing the appropriate $\chi_{h \mid t}=|t|^{-1} \int_{t} u d \Gamma$ leads to

$$
\int_{\Gamma_{C}} \psi_{h}\left(u-u_{h}\right) d \Gamma \leq C h^{\frac{3}{2}}\left\|\frac{\partial u}{\partial \boldsymbol{n}}\right\|_{H^{\frac{1}{2}\left(\Gamma_{C}\right)}}\|u\|_{H^{1}\left(\Gamma_{C}\right)} .
$$

Assembling together (6.8) and (6.9) gives the first estimate of the lemma.

In the case of the additional assumption this last term is worked out as in the proof of Lemma 4.6 modulo some slight modifications, which yields the second estimate of the lemma.

Proof of Theorem [6.5. Using Lemmas 4.1, 6.6] and 6.7] we deduce that

$$
\left\|u-u_{h}\right\|_{H^{1}(\Omega)}^{2} \leq C\left(h^{\frac{3}{2}}\|u\|_{H^{2}(\Omega)}+h\left\|u-u_{h}\right\|_{H^{1}(\Omega)}\right)\|u\|_{H^{2}(\Omega)} .
$$

Hence the result follows by Cauchy-Schwarz.

The error estimate for the Lagrange multiplier $\varphi$ follows from the inf-sup condition of Lemma 6.3 and Theorem 6.5.

Corollary 6.8. Let $(u, \varphi)$ be the solution to the mixed Signorini problem (2.13)(2.14), and $\left(u_{h}, \varphi_{h}\right) \in Y_{h}(\Omega) \times N_{h}\left(\Gamma_{C}\right)$ the solution to the discrete mixed inequality (6.2) - (6.3). Assume $u \in H^{2}(\Omega)$. Then

$$
\left\|\varphi-\varphi_{h}\right\|_{H^{-\frac{1}{2}}\left(\Gamma_{C}\right)} \leq C h^{\frac{3}{4}}\|u\|_{H^{2}(\Omega)} .
$$


Moreover, if in addition the number of points in $\Gamma_{C}$ where the constraint changes from binding to nonbinding is finite, then

$$
\left\|\varphi-\varphi_{h}\right\|_{H^{-\frac{1}{2}}\left(\Gamma_{C}\right)} \leq C h|\log (h)|^{\frac{1}{4}}\|u\|_{H^{2}(\Omega)} .
$$

Remark 6.9. When the contact zone $\Gamma_{C}$ has one (or two) common extreme point with $\Gamma_{u}$ (say $\boldsymbol{c}_{1}$ ), meaning that a Dirichlet boundary condition is enforced on $\boldsymbol{c}_{1}$, the construction of both spaces $Y_{h}(\Omega)$ and $N_{h}\left(\Gamma_{C}\right)$ does not change and the numerical analysis remains valid as is. The only modification to be taken into account is the

norm used on the Lagrange multipliers, which is in this case $\|\cdot\|_{H_{00}^{\frac{1}{2}}\left(\Gamma_{c},\left\{\boldsymbol{c}_{1}\right\}\right)^{\prime}}$ instead of $\|\cdot\|_{H^{-\frac{1}{2}}\left(\Gamma_{c}\right)}$.

\section{NumericAl DisCUSSION}

To illustrate the finite element solutions computed by the three approaches we consider the Signorini problem on the squared domain $\Omega=[0,1]^{2}$. For both experiences examined below, the unilateral side is the lower edge $\Gamma_{C}=[0,1] \times\{0\}$ and the volume data is fixed as $f=\sin (2 \pi x)$. The different simulations are run using a $\mathrm{C}++$ linear triangular finite element code developed by our team. The discrete solution is obtained as the argument of a convex minimization problem using the conjugate gradient algorithm.

Since an explicit solution of Signorini's problem is not available to us, the methodology followed for our purpose consists in calculating the discrete solution by the quadratic triangular finite elements with sufficiently high resolution $\left(h=\frac{1}{2} 56\right)$, which is then taken as the reference solution $u$. Next, we compute $u_{h}$, the approximated solution of Signorini's problem, by the conforming, nonconforming and stabilized linear finite element methods using structured meshes for mesh sizes $h \in\left\{\frac{1}{4}, \frac{1}{8}, \frac{1}{16}, \frac{1}{32}, \frac{1}{64}\right\}$, and we compare it with the reference solution. The examination of the convergence curves gives us a pertinent insight on the performances of each of the approximations, and we can draw some reliable conclusions.

In the first experiment we carried out, both vertical edges are subjected to homogeneous Neumann conditions while on the upper edge a homogeneous Dirichlet condition is prescribed. In Figure 7.1 we plotted the reference solution-actually,

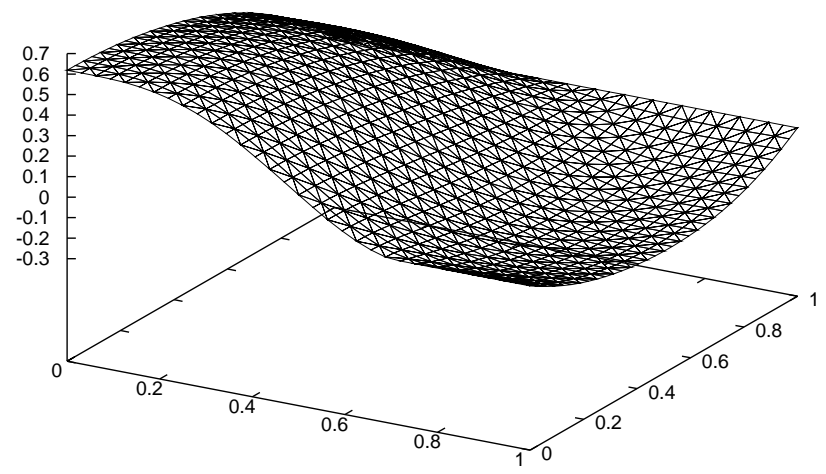

Figure 7.1. 
TABLE 7.1.

\begin{tabular}{|c|c|c|c|c|}
\hline & \multicolumn{4}{|c|}{$L^{2}$-error } \\
\hline & $h=$ & 0.0625 & 0.03125 & 0.015625 \\
\hline & $\mathrm{CFE}$ & 0.005074 & 0.00122 & 0.00030241 \\
\hline & $\mathrm{NCFE}$ & 0.005078 & 0.00123 & 0.00030246 \\
\hline & $\mathrm{SFE}$ & 0.005023 & 0.00124 & 0.00030325 \\
\hline \hline & & \multicolumn{4}{|c|}{$H^{1}$-error } \\
\hline & $\mathrm{CFE}$ & 0.0843 & 0.04169 & 0.02035 \\
\hline & $\mathrm{NCFE}$ & 0.0844 & 0.04170 & 0.02036 \\
\hline & $\mathrm{SFE}$ & 0.0842 & 0.04172 & 0.02034 \\
\hline \hline & \multicolumn{4}{|c|}{$L^{\infty}$-error } \\
\hline & $\mathrm{CFE}$ & 0.01025 & 0.0027969 & 0.0009717 \\
\hline & $\mathrm{NCFE}$ & 0.01136 & 0.0027963 & 0.0009716 \\
\hline & $\mathrm{SFE}$ & 0.01020 & 0.0025073 & 0.0007983 \\
\hline
\end{tabular}

the curve represents the interpolant of the reference solution on the coarser grid determined by $h=\frac{1}{32}$. The hypothesis made in Theorem 5.2 to obtain estimate (5.2) and in Theorem 6.5] for estimate (6.7) is clearly fulfilled: there is a single point where the constraint changes from binding to nonbinding.

Table 7.1 provides, for all the approximations (CFE, NCFE and SFE indicate the conforming, the nonconforming and the stabilized finite elements respectively)

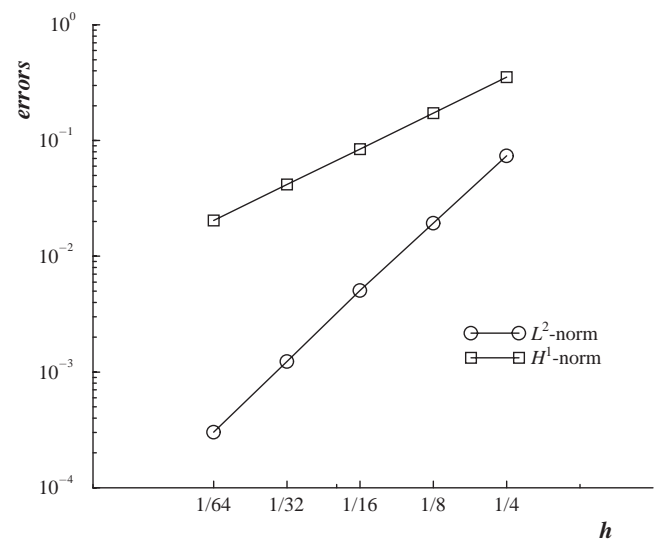

Figure 7.2. 
the variation of the $L^{2}-, H^{1}$ - and $L^{\infty}$-errors respectively with respect to the mesh sizes $h=\frac{1}{16}, \frac{1}{32}$ and $\frac{1}{64}$. The first conclusion that comes to mind is that the three methods give almost the same results with a satisfactory accuracy.

We are also interested in the convergence rate of these discretizations, mainly for the $L^{2}$ - and $H^{1}$-errors, so as to figure out whether the numerical results are in agreement with the theoretical predictions. To cope with this we choose to plot in Figure 7.2, in a logarithmic scale for both axes, the curves of these errors as functions of the mesh size $h$ only for the conforming finite elements. This is motivated by our desire not to have superposed curves, because, based on the results of Table 7.1 the nonconforming and the stabilized methods are expected to provide almost the same curves.

The evaluation of the linear regression of each curve leads to the following observations:

- The slope of the $L^{2}$-error curve is approximately 1.98 , which indicates that this error decays like $h^{2}$. This behavior is expected, although the corresponding mathematical analysis is not available.

- The slope of the $H^{1}$-error curve is calculated to 1.02 , meaning that the error decreases approximately like $h$. This is proven for the conforming (5.3) of Theorem [5.2) and for the stabilized method (6.7) of Theorem 6.5].

It is important to emphasize that the nonconforming method behaves, for this test and for the next one, in an optimal way. This makes us feel that the reason why we failed to prove optimality in this case is only of a technical origin.

The second phase of our numerical investigation is to find out whether the trends observed in the first experiment are confirmed when the extreme points of $\Gamma_{C}$ are fixed to Dirichlet data. We consider the case where the whole complementary part of $\Gamma_{C}$ is subjected to a homogeneous Dirichlet condition. Of course, the modifications detailed in Remarks 4.8 [5.5 and 6.9 have to be introduced in the construction of the discrete convex cones $K_{h}^{u}, K_{h}^{\varphi}$ and $K_{h}$.

The reference solution is depicted in Figure 7.3, where it appears that here also the constraint changes from binding to nonbinding, through only one point. Table 7.2 illustrates the equivalence of all the approaches where the approximated solution is computed using the grid corresponding to $h=\frac{1}{32}$. Finally, the plots of Figure 7.4 allow us to evaluate the different convergence rates: for the $L^{2}$-error the slope is close to 1.90 (quadratic convergence) and for the $H^{1}$-norm it is equal to 0.98 (linear convergence), which is in accordance with the theory.

TABLE 7.2.

\begin{tabular}{|c|c|c|c|}
\hline & $L^{2}$-error & $H^{1}$-error & $L^{\infty}$-error \\
\hline $\mathrm{CFE}$ & 0.00068126 & 0.040533 & 0.0023162 \\
\hline $\mathrm{NCFE}$ & 0.00068119 & 0.040557 & 0.0023148 \\
\hline $\mathrm{SFE}$ & 0.00068926 & 0.040606 & 0.0027593 \\
\hline
\end{tabular}




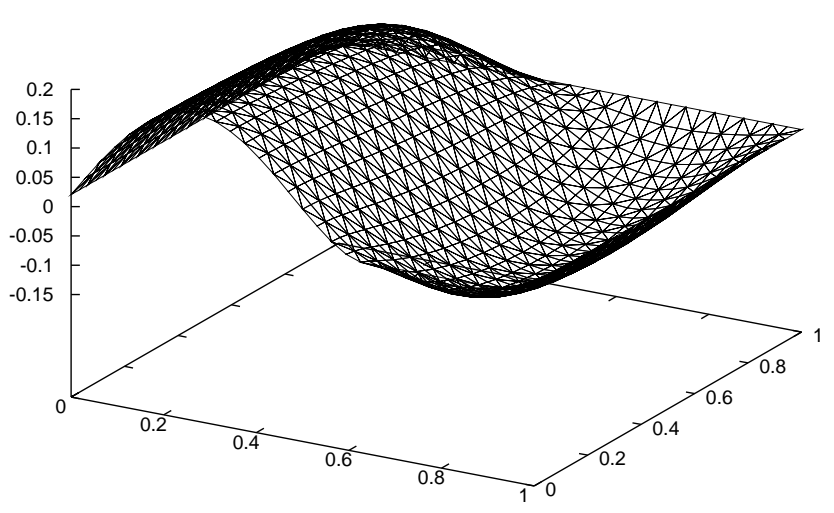

Figure 7.3.

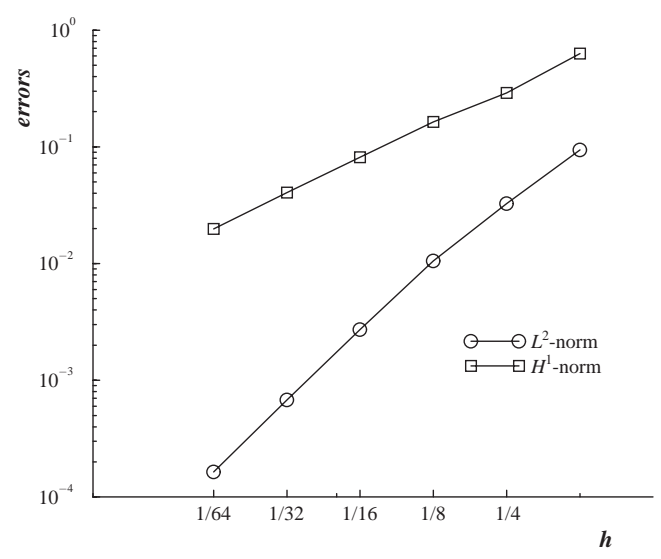

FiguRE 7.4 .

\section{Concluding Remarks}

This work is a review of the most important - to our knowledge-linear finite element approximations of the Signorini problem set in a mixed variational formulation. We develop appropriate tools to improve the convergence rates on the displacement for two discretizations (sections 4 and 5). We also derive an error estimate on the Lagrange multiplier which is the stress field on the contact region.

For the third technique a stabilization based on bubble functions is proposed and analyzed which makes it coherent for different boundary condition configurations. Conceptually this stabilized method is generalized as well to the three-dimensional problems, and the numerical analyses give similar convergence results (see [5]). Besides, it is possible to extend such an approach to consider nonmatching grids when approximation of the unilateral contact problem between two deformable solids, using the mortar concept following the ideas developed in [6] (see also [4], [21]). 


\section{Appendix A. SOME REgularized \\ INTERPOLATION OPERATOR PRESERVING SIGNS}

Our purpose is the construction of a regularization interpolation operator $r_{h}$ : $L^{2}\left(\Gamma_{C}\right) \rightarrow W_{h}\left(\Gamma_{C}\right)$ preserving the nonnegativity, i.e., if $\varphi \geq 0$, then $\left(r_{h} \varphi\right) \geq 0$, and having optimal approximation estimates for $\varphi \in H^{s}\left(\Gamma_{C}\right)\left(0 \leq s \leq \frac{1}{2}\right), \varphi \geq$ 0 . To alleviate the presentation and avoid parametrization, we identify $\Gamma_{C}$ with the segment $[0,1]$, the nodes being denoted $\left(x_{i}\right)_{0 \leq i \leq i^{*}}$ increasingly ordered, $t_{i}=$ $\left[x_{i}, x_{i+1}\right]$, with $h_{i}=\left|x_{i+1}-x_{i}\right|$ being the elements of the mesh $\mathscr{T}_{h}^{C}$ and $h=\max _{i} h_{i}$. The mesh $\mathscr{T}_{h}^{C}$ satisfies the assumption (3.4). The notation used in the sequel follows, in a large extent, that introduced in [8]. For any $i\left(0 \leq i \leq i^{*}\right)$, let $T_{i}$ denote the macro segment which is the union $t_{i-1} \cup t_{i}=\left(\xi_{i-1}, \xi_{i+1}\right)$, while $T_{i^{*}}=t_{i^{*}}$ and $T_{0}=t_{0}$ - the index $i$ in $T_{i}$ refers to the node $x_{i}$, and $T_{i}$ is then related to $x_{i}$. to the first kind of segments we associate the reference macro-interval $\hat{T}=(-1,1)$, which is a union of the reference intervals $\hat{t}^{+}=(0,1)$ and $\hat{t}^{-}=(-1,0)$, and the invertible piecewise linear mapping $F_{i}$ defined by

$$
F_{i}(\hat{\xi})= \begin{cases}\left|t_{i-1}\right| \hat{\xi}+\xi_{i} & \text { if } \xi \in \hat{t}^{-}, \\ \left|t_{i}\right| \hat{\xi}+\xi_{i} & \text { if } \xi \in \hat{t}^{+} .\end{cases}
$$

On the other hand, if $i \in\left\{0, i^{*}\right\}$, the mapping $F_{i}$ is the classical invertible linear transformation from $\hat{T}=(0,1)$ on $T_{i}$. Moreover, we need to define the interval $T_{t}$ for any $t=t_{i}$ : it is the union of $t$ and its neighbors, for instance $T_{t_{0}}=t_{0} \cup t_{1}=\left(x_{0}, x_{2}\right)$ and $T_{t_{1}}=t_{0} \cup t_{1} \cup t_{2}=\left(x_{0}, x_{3}\right)$. We begin by defining some local operators. Then, consider the local finite element spaces

$$
\begin{aligned}
W(\hat{T}) & =\left\{\hat{\psi} \in \mathscr{C}(\hat{T}), \forall \hat{t} \in \hat{T}, \quad \hat{\psi}_{\mid \hat{t}} \in \mathcal{P}_{1}(\hat{t})\right\}, \\
W_{h}\left(T_{i}\right) & =\left\{\psi_{h} \in \mathscr{C}\left(T_{i}\right), \quad \forall t \in T_{i}, \quad \psi_{h \mid t} \in \mathcal{P}_{1}(t)\right\},
\end{aligned}
$$

and the local finite element convex cones

$$
\begin{aligned}
M(\hat{T}) & =\{\hat{\psi} \in W(\hat{T}), \hat{\psi} \geq 0\}, \\
M_{h}\left(\hat{T}_{i}\right) & =\left\{\psi_{h} \in W_{h}\left(T_{i}\right), \quad \psi_{h} \geq 0\right\} .
\end{aligned}
$$

Then, for any function $\hat{\varphi} \in L^{1}(\hat{T})$ we define respectively $\hat{r}_{W}(\hat{\varphi}) \in W(\hat{T})$ and $\hat{r}_{M}(\hat{\varphi}) \in M(\hat{T})$ by

$$
\begin{array}{ll}
\forall \hat{\psi} \in W(\hat{T}), \quad & \int_{\hat{T}}\left(\hat{\varphi}-\hat{r}_{W}(\hat{\varphi})\right) \hat{\psi} d \hat{\xi}=0 \\
\forall \hat{\psi} \in M(\hat{T}), \quad & \int_{\hat{T}}\left(\hat{\varphi}-\hat{r}_{M}(\hat{\varphi})\right)\left(\hat{\psi}-\hat{r}_{M}(\hat{\varphi})\right) d \hat{\xi} \leq 0 .
\end{array}
$$

Next, let $i\left(0 \leq i \leq i^{*}\right)$ be fixed. Then for any function $\varphi \in L^{1}\left(T_{i}\right)$ we define

$$
\begin{aligned}
& r_{W}^{i}(\varphi)=\hat{r}_{W}\left(\varphi \circ F_{i}\right) \in W_{h}\left(T_{i}\right), \\
& r_{M}^{i}(\varphi)=\hat{r}_{M}\left(\varphi \circ F_{i}\right) \in M_{h}\left(T_{i}\right) .
\end{aligned}
$$

Of course there are two kinds of reference macro-elements, and the one associated with $T_{i}$ should be denoted $\hat{T}_{i}$, but we choose to drop the index $i$ of $\hat{T}_{i}$ for a while. The definition of the interpolation operator $r_{W}^{i}$ is due to Clément (see [14]) and 
is analyzed in detail in [8], and we recall the approximation result proven therein: $\forall t \in T_{i}, \forall \varphi \in H^{s}\left(\Gamma_{C}\right), s \leq 2$,

$$
\left\|\varphi-r_{W}^{i} \varphi\right\|_{L^{2}(t)} \leq C h_{i}^{s}|\varphi|_{H^{s}\left(T_{i}\right)} .
$$

The particular feature of the operator $r_{M}^{i}$ is the nonnegativity preservation, and we have

Lemma A.1. For any real number $s \in[0,2]$, there exists a constant $C=C(s)$ independent of $h$ such that for any $T_{i}\left(0 \leq i \leq i^{*}\right)$ and any $t \in T_{i}$ the following inequality holds: $\forall \varphi \in H^{s}\left(\Gamma_{C}\right), \varphi \geq 0$,

$$
\left\|\varphi-r_{M}^{i} \varphi\right\|_{L^{2}(t)} \leq C h_{i}^{s}|\varphi|_{H^{s}\left(T_{i}\right)} .
$$

Proof. Let $t_{*}$ be an interval of the macro-interval $T_{i}$. Then we have

$$
\left\|\varphi-r_{M}^{i} \varphi\right\|_{L^{2}\left(t_{*}\right)}=\left|t_{*}\right|^{\frac{1}{2}}\left\|\hat{\varphi}-\hat{r}_{M} \hat{\varphi}\right\|_{L^{2}(\hat{t})} \leq\left|t_{*}\right|^{\frac{1}{2}}\left\|\hat{\varphi}-\hat{r}_{M} \hat{\varphi}\right\|_{L^{2}(\hat{T})}
$$

As $\hat{r}_{M} M(\hat{T})$ invariant, and by the equivalence of all the norms in the finite dimensional space $W(\hat{T})$, we derive that for any $\hat{\theta} \in M(\hat{T})$

$$
\left\|\hat{\varphi}-\hat{r}_{M} \hat{\varphi}\right\|_{L^{2}(\hat{T})}=\left\|(\hat{\varphi}-\hat{\theta})-\hat{r}_{M}(\hat{\varphi}-\hat{\theta})\right\|_{L^{2}(\hat{T})} \leq \hat{c}\|(\hat{\varphi}-\hat{\theta})\|_{L^{2}(\hat{T})} .
$$

By the Bramble-Hilbert theorem we deduce that

$$
\left\|\hat{\varphi}-\hat{r}_{M} \hat{\varphi}\right\|_{L^{2}(\hat{T})} \leq \hat{c}|\hat{\varphi}|_{H^{s}(\hat{T})} .
$$

Going back to $T_{i}$ by a variable change, we have

$$
\left\|\varphi-r_{h}^{i} \varphi\right\|_{L^{2}\left(t_{*}\right)} \leq \hat{c}\left|t_{*}\right|^{\frac{1}{2}}\left(\sum_{t \in T_{i}}|t|^{2 s-1}|\varphi|_{H^{s}(t)}^{2}\right)^{\frac{1}{2}} .
$$

Using assumption (3.4) on the mesh $\mathscr{T}_{h}^{C}$ yields the result.

For $i\left(0 \leq i \leq i^{*}\right)$ let $\varphi_{i}$ denote the basis function of $W_{h}\left(\Gamma_{C}\right)$ that takes the value 1 at the point $x_{i}$ and vanishes at all other nodes. Next, for any given function $\varphi \in L^{2}\left(\Gamma_{C}\right)$ we define the regularized interpolants

$$
\tilde{r}_{h} \varphi=\sum_{i=0}^{i^{*}}\left[r_{W}^{i}(\varphi)\right]\left(x_{i}\right) \varphi_{i} \quad \text { and } \quad r_{h} \varphi=\sum_{i=0}^{i^{*}}\left[r_{M}^{i}(\varphi)\right]\left(x_{i}\right) \varphi_{i} .
$$

The operator $\tilde{r}_{h}$ is studied in [8], and the following error estimate holds true for any $s \in[0,2]: \forall \varphi \in H^{s}\left(\Gamma_{C}\right), \forall t \in \mathscr{T}_{h}^{C}$,

$$
\left\|\varphi-\tilde{r}_{h} \varphi\right\|_{L^{2}(t)} \leq C h_{i}^{s}|\varphi|_{H^{s}\left(T_{t}\right)} .
$$

Besides, we have uniform stability in $H^{\nu}\left(\Gamma_{C}\right)$ for any $\nu \in[0,1]$, meaning that for any $t \in \mathscr{T}_{h}^{C}$ and any $\varphi \in H^{\nu}\left(\Gamma_{C}\right)$,

$$
\left\|\tilde{r}_{h} \varphi\right\|_{H^{\nu}(t)} \leq C\|\varphi\|_{H^{\nu}\left(T_{t}\right)} .
$$

The regularizing operator $r_{h}$ preserves the nonnegativity, and we have the following result

Proposition A.2. Assume that the triangulation $\mathscr{T}_{h}^{C}$ fulfills the Crouzeix-Thomée criterion (3.4). Then there exists a constant $C$, independent of $h$, such that for any $t \in \mathscr{T}_{h}^{C}$ and any $s \in[0,2], \forall \varphi \in H^{s}\left(\Gamma_{C}\right), \varphi \geq 0$,

$$
\left\|\varphi-r_{h} \varphi\right\|_{L^{2}(t)} \leq C h^{s}|\varphi|_{H^{s}\left(T_{t}\right)} .
$$


Proof. Let $t=t_{i}$; then $T_{t}=\left(x_{i-1}, x_{i+2}\right)$. Following [8], we write

$$
\left(\varphi-r_{h}(\varphi)\right)_{\mid t}=\left(\varphi-r_{M}^{i+1}(\varphi)\right)_{\mid t}+\left[r_{M}^{i+1}(\varphi)-r_{M}^{i}(\varphi)\right]\left(x_{i}\right) \varphi_{i \mid t} .
$$

Estimating the first part is direct from Lemma A.1, while the second requires a technical preparation:

$$
\begin{aligned}
& \left\|\left[r_{M}^{i+1}(\varphi)-r_{M}^{i}(\varphi)\right]\left(x_{i}\right) \varphi_{i}\right\|_{L^{2}(t)} \\
& \quad=\hat{c}|t|^{\frac{1}{2}}\left\|r_{M}^{i+1}(\varphi)-r_{M}^{i}(\varphi)\right\|_{L^{\infty}(t)}=\hat{c}|t|^{\frac{1}{2}}\left\|\hat{r}_{M}^{i+1}(\varphi)-\hat{r}_{M}^{i}(\varphi)\right\|_{L^{\infty}(\hat{t})} \\
& \quad \leq \hat{c}|t|^{\frac{1}{2}}\left\|\hat{r}_{M}^{i+1}(\varphi)-\hat{r}_{M}^{i}(\varphi)\right\|_{L^{2}(\hat{t})} \leq \hat{c}\left\|r_{M}^{i+1}(\varphi)-r_{M}^{i}(\varphi)\right\|_{L^{2}(t)} .
\end{aligned}
$$

Using Lemma A.1 leads to the result.

Remark A.3. It is immediate that if $\varphi_{\mid T_{t}}=0$, then $\left(r_{h} \varphi\right)_{\mid t}=0$.

Corollary A.4. Assume that the triangulation $\mathscr{T}_{h}^{C}$ fulfills the Crouzeix-Thomée criterion (3.4). Then there exists a constant $C$, independent of $h$, such that for any $s \in[0,2], \forall \varphi \in H^{s}\left(\Gamma_{C}\right), \varphi \geq 0$,

$$
\left\|\varphi-r_{h} \varphi\right\|_{L^{2}\left(\Gamma_{C}\right)} \leq C h^{s}|\varphi|_{H^{s}\left(\Gamma_{C}\right)}
$$

Proof. This is directly obtained from (A.5) of the previous proposition.

Remark A.5. For $s>\frac{1}{2}$, it is possible to construct an interpolation operator $\tilde{r}_{h}^{0}$ : $H_{0}^{s}\left(\Gamma_{C},\left\{\boldsymbol{c}_{1}\right\}\right) \rightarrow W_{h}^{0}\left(\Gamma_{C},\left\{\boldsymbol{c}_{1}\right\}\right)$ by restricting the sum on the shape functions to the indices $i\left(1 \leq i \leq i^{*}\right)$ :

$$
\tilde{r}_{h}^{0} \varphi=\sum_{i=1}^{i^{*}}\left[r_{W}^{i}(\varphi)\right]\left(x_{i}\right) \varphi_{i}
$$

This operator satisfies similar stability conditions as (A.4) and the approximation estimate (A.5).

Remark A.6. Obviously when $s>\frac{1}{2}$, the Lagrangain interpolation operator currently denoted $i_{h}$ preserves the sign and satisfies the desired error estimates. However, when less regular functions (of $H^{s}\left(\Gamma_{C}\right), s \leq \frac{1}{2}$ ) are dealt with, we fail to define their Lagrangian interpolants. Then, the use of the regularization operators becomes necessary.

The results of Proposition A.2 may be extended to higher dimensions, in particular to two and three dimensions, following the arguments of [8].

\section{Appendix B. Analysis of a one-Dimensional FINITE ELEMENT PROJECTION OPERATOR}

The aim is to define a projection operator $\check{\pi}_{h}: L^{2}\left(\Gamma_{C}\right) \rightarrow \tilde{M}_{h}\left(\Gamma_{C}\right)$ satisfying optimal approximation results with respect to the $H_{00}^{\frac{1}{2}}\left(\Gamma_{C},\left\{\boldsymbol{c}_{1}\right\}\right)^{\prime}$-norm and so that $\check{\pi}_{h} \varphi$ is in the convex cone $M_{h}^{u}\left(\Gamma_{C}\right)([5.6)$ if $\varphi \geq 0$, which means that

$$
\int_{\Gamma_{C}} \check{\pi}_{h} \varphi \chi_{h} d \Gamma \geq 0, \quad \forall \chi_{h} \in W_{h}^{0}\left(\Gamma_{C},\left\{\boldsymbol{c}_{1}\right\}\right), \chi_{h} \geq 0 .
$$

Such an operator is used to derive an error estimate on the Lagrange multiplier $\varphi$ in Remark 5.5

Consider the following definition: $\forall \psi \in L^{2}\left(\Gamma_{C}\right)$,

$$
\int_{\Gamma_{C}}\left(\varphi-\check{\pi}_{h} \varphi\right) \psi_{h} d \Gamma=0, \quad \forall \psi_{h} \in W_{h}^{0}\left(\Gamma_{C},\left\{\boldsymbol{c}_{1}\right\}\right) .
$$


Obviously, as soon as $\varphi \geq 0$ the $\check{\pi}_{h} \varphi$ belongs to $M_{h}^{u}\left(\Gamma_{C}\right)$. Furthermore, we have

Lemma B.1. The following approximation holds: $\forall \varphi \in H^{\frac{1}{2}}\left(\Gamma_{C}\right)$,

$$
\left\|\varphi-\check{\pi}_{h} \varphi\right\|_{H_{00}^{\frac{1}{2}}\left(\Gamma_{C},\left\{\boldsymbol{c}_{1}\right\}\right)^{\prime}} \leq C h\|\varphi\|_{H^{\frac{1}{2}}\left(\Gamma_{C}\right)} .
$$

Proof. As in Appendix A, we identify $\Gamma_{C}$ with the segment $[0,1]$, with the same notation introduced there. Let us define the function

$$
\alpha_{\mid t_{1}}(x)=\frac{x-x_{0}}{x_{1}-x_{0}}, \quad \text { and } \quad \alpha_{\mid t_{i}}(x)=1, \quad \forall i \geq 2 .
$$

Then, for any $\varphi \in L^{2}\left(\Gamma_{C}\right)$, choosing $\chi_{h}=\alpha\left(\check{\pi}_{h} \varphi\right) \in W_{h}^{0}\left(\Gamma_{C},\left\{\boldsymbol{c}_{1}\right\}\right)$ in (B.1) produces

$$
\left\|\sqrt{\alpha}\left(\check{\pi}_{h} \varphi\right)\right\|_{L^{2}\left(\Gamma_{C}\right)} \leq\|\sqrt{\alpha} \varphi\|_{L^{2}\left(\Gamma_{C}\right)} \leq\|\varphi\|_{L^{2}\left(\Gamma_{C}\right)},
$$

from which we deduce the preliminary result: $\forall \varphi \in H^{\frac{1}{2}}\left(\Gamma_{C}\right)$

$$
\left\|\sqrt{\alpha}\left(\varphi-\check{\pi}_{h} \varphi\right)\right\|_{L^{2}\left(\Gamma_{C}\right)} \leq 2 \inf _{\psi_{h} \in \tilde{M}_{h}\left(\Gamma_{C}\right)}\left\|\varphi-\psi_{h}\right\|_{L^{2}\left(\Gamma_{C}\right)} \leq C h^{\frac{1}{2}}\|\varphi\|_{H^{\frac{1}{2}}\left(\Gamma_{C}\right)} .
$$

By duality it can be written

$$
\left\|\varphi-\check{\pi}_{h} \varphi\right\|_{H_{00}^{\frac{1}{2}}\left(\Gamma_{C},\left\{c_{1}\right\}\right)^{\prime}}=\sup _{\chi \in H_{00}^{\frac{1}{2}}\left(\Gamma_{C},\left\{c_{1}\right\}\right)} \frac{1}{\|\chi\|_{H_{00}^{\frac{1}{2}}\left(\Gamma_{C},\left\{c_{1}\right\}\right)}} \int_{\Gamma_{C}}\left(\varphi-\check{\pi}_{h} \varphi\right) \chi d \Gamma
$$

Then, as allowed by the definition of $\check{\pi}_{h}$, we have, $\forall \chi_{h} \in W_{h}^{0}\left(\Gamma_{C},\left\{\boldsymbol{c}_{1}\right\}\right)$,

$$
\begin{aligned}
\int_{\Gamma_{C}}\left(\varphi-\check{\pi}_{h} \varphi\right) \chi d \Gamma & =\int_{\Gamma_{C}}\left(\varphi-\check{\pi}_{h} \varphi\right)\left(\chi-\chi_{h}\right) d \Gamma \\
& \leq\left\|\sqrt{\alpha}\left(\varphi-\check{\pi}_{h} \varphi\right)\right\|_{L^{2}\left(\Gamma_{C}\right)}\left\|\frac{\chi-\chi_{h}}{\sqrt{\alpha}}\right\|_{L^{2}\left(\Gamma_{C}\right)} .
\end{aligned}
$$

Handling the term on $\chi$, we get

$$
\begin{aligned}
\left\|\frac{\chi-\chi_{h}}{\sqrt{\alpha}}\right\|_{L^{2}\left(\Gamma_{C}\right)}^{2} & =\sum_{i \geq 2}\left\|\chi-\chi_{h}\right\|_{L^{2}\left(t_{i}\right)}^{2}+\left\|\frac{\chi-\chi_{h}}{\sqrt{\alpha}}\right\|_{L^{2}\left(t_{1}\right)}^{2} \\
& =\sum_{i \geq 2}\left\|\chi-\chi_{h}\right\|_{L^{2}\left(t_{i}\right)}^{2}+h_{1}\left\|\frac{\chi-\chi_{h}}{\sqrt{x-x_{0}}}\right\|_{L^{2}\left(t_{1}\right)}^{2} \\
& \leq \sum_{i \geq 2}\left\|\chi-\chi_{h}\right\|_{L^{2}\left(t_{i}\right)}^{2}+h_{1}\left\|\chi-\chi_{h}\right\|_{H_{00}}^{2}{ }_{\frac{1}{2}\left(t_{1},\left\{\boldsymbol{c}_{1}\right\}\right)} .
\end{aligned}
$$

Then, constructing $\chi_{h}=\tilde{r}_{h}^{0} \chi$ as in Remark A.3, we obtain (B.6)

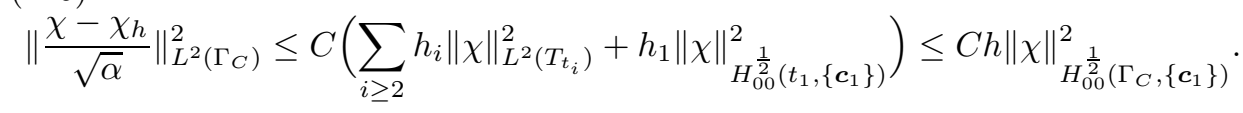

Going back to (B.5) and using (B.3) and (B.6) yields

$$
\int_{\Gamma_{C}}\left(\varphi-\check{\pi}_{h} \varphi\right) \chi d \Gamma \leq C h\|\varphi\|_{H^{\frac{1}{2}}\left(\Gamma_{C}\right)}\|\chi\|_{H_{00}^{\frac{1}{2}}\left(\Gamma_{C},\left\{\boldsymbol{c}_{1}\right\}\right)} .
$$

The duality (B.4) ends the proof. 


\section{REFERENCES}

1. R. A. Adams. Sobolev Spaces, Academic Press, 1975. MR 56:9247

2. F. Ben Belgacem. Discrétisations 3D non conformes par la méthode de décomposition de domaine des éléments avec joints : Analyse mathématique et mise en ouvre pour le problème de Poisson. Thèse de l'Université Pierre et Marie Curie, Paris VI. Note technique EDF, ref. HI72/93017 (1993).

3. F. Ben Belgacem. Numerical Simulation of some Variational Inequalities Arisen from Unilateral Contact Problems by the Finite Element Method, SIAM J. Numer. Anal., 37: 1198-1216, 2000. MR 2002c:74066

4. F. Ben Belgacem. The Mortar Finite Element Method with Lagrange Multipliers, Numer. Math. 84, 173-197, 1999. MR 2000k:65204

5. F. Ben Belgacem ans S. C. Brenner. Some Nonstandard Finite Element Estimates with Applications to 3D Poisson and Signorini Problems, Electronic Transactions in Numerical Analysis, 12: 134-148, 200. MR 2002c: 65187

6. F. Ben Belgacem, P. Hild and P. Laborde. Extension of the Mortar Finite Element to a Variational Inequality Modeling Unilateral Contact, Math. Models and Methods in Appl. Sci., 9: 287-303, 1999. MR 99m:73066

7. C. Bernardi, N. Débit and Y. Maday. Coupling Finite Element and Spectral Methods: First Results, Math. Comp., 54, 21-39, 1990. MR 90f:65201

8. C. Bernardi and V. Girault. A Local Regularisation Operator for Triangular and Quadrangular Finite Elements, SIAM J. Numer. Anal., 35, 1893 - 1916, 1998. MR 99g:65107

9. C. Bernardi, Y. Maday et A. T. Patera. A New Non Conforming Approach to Domain Decomposition: The Mortar Element Method. Collège de France Seminar, Pitman, H. Brezis, J.-L. Lions, 1990. MR 95a:65201

10. F. Brezzi, L. P. Franca, D. Marini and A. Russo. Stabilization Techniques for Domain Decomposition with Nonmatching Grids, Domain Decomposition Methods in Sciences and Engineering, (P. Bjrstad, M. Espedal, D. Keyes, Eds.), Domain Decomposition Press, Bergen, $1-11,1998$, (IX International Conference, Bergen, Norway, June 1996).

11. F. Brezzi, W. W. Hager and P. A. Raviart. Error estimates for the finite element solution of variational inequalities, Numer. Math., 28, 431-443, 1977. MR 56:7254

12. F. Brezzi and D. Marini. Error Estimates for the Three-field Formulation with Bubble Stabilization, Math. Comp., 70, 911-934, 2001. MR 2002b:65159

13. P.-G. Ciarlet. The Finite Element Method for Elliptic Problems, North Holland, 1978. MR 58:25001

14. P. Clément. Approximation by Finite Elements Functions Using Local Regularization, RAIRO, Anal. Numér., 9, 77-84, 1975. MR 53:4569

15. P. Coorevits, P. Hild, K. Lhalouani and T. Sassi. Mixed Finite Element Method for Unilateral Problem: Convergence Analysis and Numerical Studies, Math. Comp., 71, 1-25, 2002. MR 2002g:74043

16. M. Crouzeix and V. Thomée. The Stability in $L^{p}$ and $W^{1, p}$ of the $L^{2}$-Projection on Finite Element Function Spaces, Math. Comp., 48 521-532, 1987. MR 88f:41016

17. G. Duvaut and J.-L. Lions. Les inéquations en mécanique et en physique, Dunod, 1972. MR 57:4778

18. R. S. Falk. Error Estimates for the Approximation of a Class of Variational Inequalities, Math. Comp., 28: 963-971, 1974. MR 52:12323

19. R. Glowinski, J.-L. Lions and R. Trémolières. Analyse numériques des inéquations variationnelles, Tome 1, Dunod, 1976. MR 58:31697a

20. P. Grisvard. Elliptic Problems in Nonsmooth Domains, Monographs and Studies in Mathematics, 24, Pitman, 1985. MR 86m:35044

21. J. Haslinger and I. Hlaváček. Contact between Elastic Bodies -2.Finite Element Analysis, Aplikace Matematiky, 26, 263-290, 1981. MR 83c:73052a

22. J. Haslinger, I. Hlaváček and J. Nečas. Numerical Methods for Unilateral Problems in Solid Mechanics, in Handbook of Numerical Analysis, Vol. IV , Part 2, Eds. P.G. Ciarlet and J.-L. Lions, North Holland, 1996. MR 98b:65001

23. P. Hild. Problèmes de contact unilatéral et maillages incompatibles, Thèse de l'Université Paul Sabatier, Toulouse 3, 1998. 
24. N. Kikuchi and J. T. Oden. Contact Problems in Elasticity: A Study of Variational Inequalities and Finite Element Methods, SIAM, 1988. MR 89j:73097]

25. D. Kinderlehrer and G. Stamppachia. An Introduction to Variational Inequalities and their Applications, Academic Press, 1980. MR 81g:49013

26. K. Lhalouani and T. Sassi. Nonconforming Mixed Variational Formulation and Domain Decomposition for Unilateral Problems, East West J. Numer. Math., 7: 23-30, 1999. MR 2000b:65217

27. J.-L. Lions and E. Magenes. Problèmes aux limites non homogènes, Dunod, 1968. MR 40:512, MR 40:513

28. M. Moussaoui and K. Khodja. Régularité des solutions d'un problème mêlé Dirichlet-Signorini dans un domaine polygonal plan, Commun. Part. Diff. Eq., 17: 805-826, 1992. MR 93b:35055

29. P.-A. Raviart and J.-M. Thomas. Primal Hybrid Finite Element Methods for 2nd Order Elliptic Equations, Math. Comp., 31, 391-396, 1977. MR [55:4747

30. P. Seshaiyer and M. Suri. Uniform $h-p$ Convergence Results for the Mortar Finite Element Method, Math. Comp., 69, 521-546, 2000. MR 2000i:65192

31. G. Strang and G. J. Fix. An Analysis of the Finite Element Method, Prentice-Hall, Englewood Cliffs, NJ, 1973. MR 56:1747

32. J.-M. Thomas. Sur l'analyse numérique des méthodes d'éléments finis hybrides et mixtes. Thèse (1977), Université Pierre et Marie Curie, PARIS VI.

33. Z.-H. Zhong. Finite Element Procedures for Contact-Impact Problems, Oxford University Press, 1993.

Mathématiques pour L'Industrie et la Physique, (UMR 5640), Université Paul Sabatier, 118 route de Narbonne, 31062 Toulouse Cedex 04, France

E-mail address: belgacem@mip.ups-tlse.fr

Mathématiques pour l'Industrie et la Physique, (UMR 5640), Institut National des Sciences Appliquées, Département de Mathématique, 135 Avenue de Rangueil, 31077 Toulouse Cedex 04, France

E-mail address: Yves.Renard@gmm.insa-tlse.fr 